\title{
A differentiable homotopy to compute nash equilibria of $\mathbf{n}$-person games
}

Citation for published version (APA):

Herings, P. J. J., \& Peeters, R. J. A. P. (2000). A differentiable homotopy to compute nash equilibria of $n$ person games. METEOR, Maastricht University School of Business and Economics. METEOR Research Memorandum No. 033 https://doi.org/10.26481/umamet.2000033

Document status and date:

Published: 01/01/2000

DOI:

10.26481/umamet.2000033

Document Version:

Publisher's PDF, also known as Version of record

\section{Please check the document version of this publication:}

- A submitted manuscript is the version of the article upon submission and before peer-review. There can be important differences between the submitted version and the official published version of record.

People interested in the research are advised to contact the author for the final version of the publication, or visit the DOI to the publisher's website.

- The final author version and the galley proof are versions of the publication after peer review.

- The final published version features the final layout of the paper including the volume, issue and page numbers.

Link to publication

\footnotetext{
General rights rights.

- You may freely distribute the URL identifying the publication in the public portal. please follow below link for the End User Agreement:

www.umlib.nl/taverne-license

Take down policy

If you believe that this document breaches copyright please contact us at:

repository@maastrichtuniversity.nl

providing details and we will investigate your claim.
}

Copyright and moral rights for the publications made accessible in the public portal are retained by the authors and/or other copyright owners and it is a condition of accessing publications that users recognise and abide by the legal requirements associated with these

- Users may download and print one copy of any publication from the public portal for the purpose of private study or research.

- You may not further distribute the material or use it for any profit-making activity or commercial gain

If the publication is distributed under the terms of Article $25 \mathrm{fa}$ of the Dutch Copyright Act, indicated by the "Taverne" license above, 


\title{
A Differentiable Homotopy to Compute Nash Equilibria of $n$-Person Games
}

\author{
P. Jean-Jacques Herings* $\quad$ Ronald Peeters ${ }^{\dagger}$
}

21st January 2000

\begin{abstract}
The literature on the computation of Nash equilibria in $n$-person games is dominated by simplicial methods. This paper is the first to introduce a globally convergent algorithm that fully exploits the differentiability present in the problem. It presents an everywhere differentiable homotopy to do the computations. The homotopy path can therefore be followed by several numerical techniques. Moreover, instead of computing some Nash equilibrium, the algorithm is constructed in such a way that it computes the Nash equilibrium selected by the tracing procedure of Harsanyi and Selten. As a by-product of our proofs it follows that for a generic game the tracing procedure defines a unique feasible path. The numerical performance of the algorithm is illustrated by means of several examples.
\end{abstract}

JEL classification: C63, C72.

Keywords: Computation of equilibria; Noncooperative game theory; Tracing procedure.

\section{Introduction}

During the past few years, many research fields in which conflicts between agents arise have benefitted from the introduction of game theoretic tools. A further use of game theory may stagnate for several reasons. One is that there may be a great number of solutions to a given game. Recent work of McLennan (1999) shows that the number of Nash equilibria in normal form games of modest size is huge on average. For instance, the mean number of Nash equilibria in a game with 4 agents, each having 6 strategies is estimated to be 2.037 ,

*Department of Economics, Universiteit Maastricht, P.O. Box 616, 6200 MD Maastricht, The Netherlands. E-mail: P.Herings@algec.unimaas.nl

${ }^{\dagger}$ Department of Economics, Universiteit Maastricht, P.O. Box 616, 6200 MD Maastricht, The Netherlands. E-mail: R.Peeters@algec.unimaas.nl 
with a standard error of the estimation of 66 . Nevertheless, computation of just a single Nash equilibrium may prove to be hard in many games of interest.

This point is expressed as follows in van Damme (1995): "In the last two decades, game theoretic methods have become more and more important in economics and the other social sciences. Many scientific papers in these areas have the following basic structure: A problem is modeled as a game, the game is analyzed by computing its equilibria, and the properties of the latter are translated back into insights relevant to the original problem. ... It has been found that the tools may not be powerful enough ... For example, many models admit a vast multiplicity of equilibrium outcomes so that the predictive power of game theoretic analysis is limited. To increase understanding, it may, hence, be necessary to perfect the tools.".

A tool to increase understanding has been suggested in Harsanyi and Selten (1988), who introduce the linear tracing procedure as a means for equilibrium selection in finite $n$-person games. The linear tracing procedure is a mathematical construct that adjusts arbitrary prior beliefs into equilibrium beliefs. First, the players optimize by playing best responses against identical prior beliefs concerning the play of the other players. Next, they observe that their beliefs are not met and they subsequently update their beliefs and react optimally there upon. This updating of beliefs continues until equilibrium beliefs for the game have been found. Although the term "procedure" suggests a numerical approach, the tracing procedure itself is a non-constructive method. This brings us to a second problem, the difficulties involved in solving games of interest, which may hamper a further progress in the applications of game theory. We fully agree to the view expressed in Judd (1997) that computational methods will serve an important role in the further development of economic theory.

The aim of this paper is to present an algorithm to compute a Nash equilibrium for an arbitrary finite $n$-person game in normal form. Since there is a vast multiplicity of Nash equilibria in many games, we also find it essential to provide an algorithm that performs the task of equilibrium selection. In particular, we want to have an algorithm that computes the Nash equilibrium selected by the linear tracing procedure. Finally, the algorithm should be fast in that it allows for the computation of a Nash equilibrium for non-trivial games within reasonable time limits.

The first procedures for finding an equilibrium in $n$-person games were developed simultaneously and independently by Rosenmüller (1971) and Wilson (1971). Both methods are generalizations of the procedure of Lemke and Howson (1964) for bimatrix games. Although these methods are not directly suitable for computational purposes, their common result, the existence of a non-linear path leading to an equilibrium, was a very important 
step towards an implementable algorithm as developed by Garcia, Lemke and Lüthi (1973). Later, a more efficient algorithm was proposed in van der Laan, Talman and van der Heijden (1987). A problem of these algorithms is that they calculate only an approximation of a sample Nash equilibrium and do not bother about the game-theoretic underpinning of the calculated equilibrium.

The case of 2-person games is special in that the exact calculation of a Nash equilibrium is possible due to the linear structure of such a game. For this class of games, the algorithm of van den Elzen and Talman (1999) computes the Nash equilibrium selected by the linear tracing procedure. The generalization to $n$-person games is covered by the algorithm presented in Herings and van den Elzen (1998). That algorithm, as well as all the other algorithms discussed above, is a simplicial method. Simplicial methods solve a non-linear equilibrium problem by solving a piecewise linear approximation of the problem. All known algorithms that have been shown to converge to a Nash equilibrium for a generic $n$-person game, are simplicial methods. A drawback of these methods is that they do not exploit the differentiable structure that is present in $n$-person games. For an excellent survey of methods to compute Nash equilibria in games, we refer to McKelvey and McLennan (1996).

In this paper we propose an algorithm that is shown to converge to a Nash equilibrium for a generic $n$-person game. This algorithm is the first to exploit the differentiability present in games to the full extent. The algorithm also solves the equilibrium selection problem in that it computes the Nash equilibrium selected by the linear tracing procedure.

The algorithm belings to the class of homotopy methods. The formulation as a differentiable homotopy makes it possible to apply standard path-following techniques that are available in professionally programmed software. This makes implementation on a computer an easy exercise. As a by-product of the proof that the algorithm converges for a generic game, we obtain an elegant and clean proof of the theorem in Harsanyi (1975) that for a generic game the linear tracing procedure yields a path leading to a unique Nash equilibrium.

The paper has been organized as follows. Some notations and the definition of the linear tracing procedure are given in Section 2. In Section 3, the properties of the linear tracing procedure are studied. It is shown that for almost every game, the linear tracing procedure is formed by a finite union of arcs and loops. Using a well-chosen transformation of variables, the linear tracing procedure is described by the zeros of an everywhere differentiable homotopy function in Section 4. Section 5 discusses the implementation of the homotopy algorithm and gives some numerical results for randomly generated games. 


\section{The linear tracing procedure}

A finite $n$-person noncooperative game in normal form is a tuple $\Gamma=\left\langle N,\left\{S^{i}\right\}_{i \in N},\left\{u^{i}\right\}_{i \in N}\right\rangle$, with $S^{i}$ and $u^{i}: S \rightarrow \mathbb{R}$ the set of pure strategies and the payoff function of player $i$, respectively. The set of players is denoted by $N=\{1, \ldots, n\} . S=S^{1} \times \cdots \times S^{n}$ denotes the set of pure strategy combinations. Player $i$ has $m^{i}$ pure strategies. The total number of pure strategies is given by $m^{*}=\sum_{i \in N} m^{i}$; the total number of pure strategy combinations is given by $m=\prod_{i \in N} m^{i}$. We number the strategies of the players such that pure strategy $j \in\left\{1, \ldots, m^{i}\right\}$ of player $i$ is denoted by $s_{j}^{i}$. The union of the pure strategies over all players is given by $S^{*}=\bigcup_{i \in N} S^{i}$.

A mixed strategy of player $i$ is a probability distribution on $S^{i}$. We identify the set of all probability distributions on $S^{i}$ with $\Sigma^{i}=\left\{\sigma^{i} \in \mathbb{R}_{+}^{m^{i}} \mid \sum_{j=1}^{m^{i}} \sigma_{j}^{i}=1\right\}$. For $\sigma^{i} \in \Sigma^{i}$, the probability assigned to pure strategy $s_{j}^{i}$ is given by $\sigma_{j}^{i}$. The strategy space of the game is therefore equal to $\Sigma=\Sigma^{1} \times \cdots \times \Sigma^{n}$. Note that the dimension of $\Sigma$ equals $m^{*}-n$. Given a mixed strategy combination $\sigma \in \Sigma$ and a strategy $\bar{\sigma}^{i} \in \Sigma^{i}$, we denote by $\left(\sigma^{-i}, \bar{\sigma}^{i}\right)$ the mixed strategy that results from replacing $\sigma^{i}$ by $\bar{\sigma}^{i}$. If a mixed strategy combination $\sigma \in \Sigma$ is played, then the probability $\sigma(s)$ that the pure strategy combination $s=\left(s_{j^{1}}^{1}, \ldots, s_{j^{n}}^{n}\right)$ occurs, is given by $\sigma(s)=\prod_{i \in N} \sigma_{j^{i}}^{i}$ and the expected payoff of player $i$ by $u^{i}(\sigma)=\sum_{s \in S} \sigma(s) u^{i}(s)$. The class of all noncooperative games is denoted by $\mathcal{G}$.

A mixed strategy combination $\sigma \in \Sigma$ is said to be a Nash equilibrium of game $\Gamma$ if $\sigma^{i}$ is a best response against $\sigma^{-i}$ for all $i \in N$. The set of Nash equilibria of game $\Gamma$ is denoted by $\mathrm{NE}(\Gamma)$.

The equilibrium selection theory as presented in Harsanyi and Selten (1988) has the nice feature that it selects a unique Nash equilibrium for every game. The HarsanyiSelten theory is to a large extent based on the linear tracing procedure, a mathematical procedure introduced in Harsanyi (1975) that yields a unique Nash equilibrium for almost every game. To select the unique Nash equilibrium for the remaining measure zero set of games, Harsanyi and Selten use a construct called the logarithmic tracing procedure. The linear tracing procedure is used repeatedly in their equilibrium selection theory to find a unique solution of so-called basic games and to define risk-dominance relationships between Nash equilibria.

The linear tracing procedure models a process of convergent expectations by which rational players will come to adopt, and expect each other to adopt, a particular Nash equilibrium as a solution for a given game. Before applying the tracing procedure, every player is assumed to have a subjective probability distribution expressing his expectation about the strategic choices of the other players. Each player is assumed to use the same 
theory to determine his subjective probability distributions, which makes that all players have the same expectations about the other players. This common subjective probability distribution is called the prior. In the naive Bayesian approach, all players choose best responses to their priors and would in this way reach a strategy-combination that does not constitute a Nash equilibrium in general. In the linear tracing procedure, the information on the best responses is only gradually fed back into the expectations of the players. As the linear tracing procedure proceeds, both the priors and their best responses will gradually change until both converge to some Nash equilibrium of the game.

Each generically convergent algorithm to compute a Nash equilibrium can be interpreted as a procedure that selects an equilibrium, but will in fact be a completely arbitrary selection procedure. Harsanyi-Selten's procedure, on the other hand, is comprehensively studied and finds its origin in the search for an equilibrium selection method. Therefore, it is attractive to have an algorithm which computes the equilibrium selected by the Harsanyi-Selten theory.

Consider some $n$-person game $\Gamma$ and some prior $p \in \Sigma$. For every $t \in[0,1]$, the linear tracing procedure generates a Nash equilibrium of the game $\Gamma^{t}=\left\langle N,\left\{S^{i}\right\}_{i \in N},\left\{v^{i}(t)\right\}_{i \in N}\right\rangle$, where the payoff function $v^{i}(t): \Sigma \rightarrow \mathbb{R}$ of player $i$ is defined by

$$
v^{i}(t, \sigma)=t u^{i}(\sigma)+(1-t) u^{i}\left(p^{-i}, \sigma^{i}\right)
$$

The game $\Gamma^{0}$ corresponds to a trivial game, where all players believe that all their opponents play with probability 1 according to the prior belief. The game $\Gamma^{1}$ coincides with the original game $\Gamma$. A best response against a strategy combination $\sigma \in \Sigma$ in the game $\Gamma^{t}$ corresponds to a best response against the probability distribution $t[\sigma]+(1-t)[p]$ on $S$ in the game $\Gamma$. The latter probability distribution does in general not belong to $\Sigma$, since it may be correlated.

The linear tracing procedure links a Nash equilibrium of the game $\Gamma^{0}$ to a Nash equilibrium of $\Gamma^{1}$. The set of all Nash equilibria related to the games $\Gamma^{t}, t \in[0,1]$, is denoted by

$$
\mathcal{L}(\Gamma, p)=\left\{(t, \sigma) \in[0,1] \times \Sigma \mid \sigma \in \mathrm{NE}\left(\Gamma^{t}\right)\right\} .
$$

The linear tracing procedure is said to be feasible if there exists a path in $\mathcal{L}(\Gamma, p)$ connecting a best response against the prior to a Nash equilibrium of the game $\Gamma$, i.e. there exists a continuous function $\gamma:[0,1] \rightarrow \mathcal{L}(\Gamma, p)$ such that $\gamma(0) \in \mathcal{L}(\Gamma, p) \cap(\{0\} \times \Sigma)$ and $\gamma(1) \in \mathcal{L}(\Gamma, p) \cap(\{1\} \times \Sigma)$. In general there may be many trajectories $\gamma([0,1])$ that link a Nash equilibrium of $\Gamma^{0}$ to a Nash equilibrium of $\Gamma^{1}$. If this trajectory is unique, then 
the linear tracing procedure is said to be well-defined. If the linear tracing procedure is well-defined, then it selects a unique Nash equilibrium of the game $\Gamma$.

Schanuel, Simon and Zame (1991) have shown that the logarithmic tracing procedure connects the best responses to the prior beliefs to exactly one Nash equilibrium. From this result, the feasibility of the linear tracing procedure follows by an easy limit argument. The proofs related to the logarithmic tracing procedure use heavy mathematical machinery from the field of algebraic geometry. Herings (1997b) gives two very short proofs of the feasibility of the linear tracing procedure that do not involve the logarithmic tracing procedure.

The linear tracing procedure is shown to be well-defined for a generic $n$-person game by Harsanyi (1975). His result is that given any prior distribution almost all $n$-person noncooperative games give rise to a well-defined linear tracing procedure. As a by-product of the convergence proof for our algorithm, we obtain a clean and elegant proof of Harsanyi's result.

\section{A Piecewise Differentiable Approach}

From the previous section we know that the linear tracing procedure contains a feasible path. The strategy of our algorithm is to end up in a Nash equilibrium of the game by following that path. A simplicial method that needs very little structure of the path is implemented in Herings and van den Elzen (1998). We would like to use path following methods that exploit the differentiability of the path. Unfortunately, the feasible path displays serious non-differentiabilities for most games, which makes the computation of Nash equilibria so difficult. This section analyzes the differentiability properties of $\mathcal{L}(\Gamma, p)$ and reveals that, although not necessarily everywhere differentiable, it has a lot of structure.

Let a subset $B^{*}$ of $S^{*}$ be given with the property that for every player $i$ there is at least one pure strategy $s_{j}^{i}$ in $B^{*}$, so $B^{*} \cap S^{i} \neq \emptyset$ for every player $i$. Such a set $B^{*}$ is called admissible. The sets $B^{*}$ are used to decompose $\mathcal{L}(\Gamma, p)$ in subsets $\mathcal{L}\left(\Gamma, p, B^{*}\right)$, each having a differentiable manifold structure. The set $\mathcal{L}\left(\Gamma, p, B^{*}\right)$ contains those elements of $\mathcal{L}(\Gamma, p)$ where only strategies in $B^{*}$ are played with positive probability. It is defined by

$$
\begin{aligned}
& \mathcal{L}\left(\Gamma, p, B^{*}\right)=\{(t, \sigma) \in \mathcal{L}(\Gamma, p) \mid s_{j}^{i} \notin B^{*} \Rightarrow \sigma_{j}^{i}=0 \\
&\left.s_{j}^{i} \in B^{*} \Rightarrow s_{j}^{i} \in \operatorname{argmax}_{s_{\ell}^{i} \in S^{i}} v^{i}\left(t, \sigma^{-i}, s_{\ell}^{i}\right)\right\} .
\end{aligned}
$$

It follows that

$$
\mathcal{L}(\Gamma, p)=\bigcup_{B^{*}} \mathcal{L}\left(\Gamma, p, B^{*}\right) .
$$


Two sets $\mathcal{L}\left(\Gamma, p, B^{*}\right)$ and $\mathcal{L}\left(\Gamma, p, \bar{B}^{*}\right)$ can only have a point $(t, \sigma)$ in common if there is a player $i$ and a strategy $s_{j}^{i}$ such that $\sigma_{j}^{i}=0$ and $s_{j}^{i} \in \operatorname{argmax}_{s_{\ell}^{i} \in S^{i}} v^{i}\left(t, \sigma^{-i}, s_{\ell}^{i}\right)$, so $s_{j}^{i}$ is a best response to $\sigma^{-i}$ that is played with probability zero.

To analyze the structure of $\mathcal{L}(\Gamma, p)$ and the sets $\mathcal{L}\left(\Gamma, p, B^{*}\right)$, we design systems of equalities and inequalities whose solutions characterize these sets. The Kuhn-Tucker firstorder conditions, which provide necessary and sufficient conditions for $\sigma^{i}$ to be a best response against $\bar{\sigma}^{-i}$ in the game $\Gamma^{\bar{t}}$, are given by

$$
\begin{aligned}
& v^{i}\left(\bar{t}, \bar{\sigma}^{-i}, s_{j}^{i}\right)+\lambda_{j}^{i}-\mu^{i}=0, \quad s_{j}^{i} \in S^{i}, \\
& \lambda_{j}^{i} \geq 0, \quad \sigma_{j}^{i} \geq 0, \quad \lambda_{j}^{i} \sigma_{j}^{i}=0, \quad s_{j}^{i} \in S^{i}, \\
& \sum_{j=1}^{m^{i}} \sigma_{j}^{i}-1=0 .
\end{aligned}
$$

Here, $\lambda_{j}^{i}$ is the shadowprice of playing strategy $s_{j}^{i}$, i.e. the disutility of a marginal increase in the probability $\sigma_{j}^{i}$ by which pure strategy $s_{j}^{i}$ is played. If $\sigma_{j}^{i}>0$, then $s_{j}^{i}$ is a best response to $\bar{\sigma}^{-i}$ in game $\Gamma^{\bar{t}}$, which implies $\lambda_{j}^{i}=0$ because of the complementarity of $\lambda_{j}^{i}$ and $\sigma_{j}^{i}$. It follows that $\mu^{i}$ is equal to the payoff of player $i$ in game $\Gamma^{\bar{t}}$. The last equality, $\sum_{j=1}^{m^{i}} \sigma_{j}^{i}-1=0$, makes sure that $\sigma^{i}$ is a member of $\Sigma^{i}$.

Given an admissible subset $B^{*}$, we define $\mathcal{O}\left(\Gamma, p, B^{*}\right)$ as the set of solutions $(t, \sigma, \lambda, \mu)$ to the following equalities and inequalities:

(1) $\quad v^{i}\left(t, \sigma^{-i}, s_{j}^{i}\right)+\lambda_{j}^{i}-\mu^{i}=0, \quad s_{j}^{i} \in S^{*}$,

(2) $\quad \sigma_{j}^{i}=0, \quad s_{j}^{i} \notin B^{*}$,

(3) $\lambda_{j}^{i}=0, \quad s_{j}^{i} \in B^{*}$,

(4) $\quad \sum_{j=1}^{m^{i}} \sigma_{j}^{i}-1=0, \quad i \in N$,

(5) $\sigma_{j}^{i} \geq 0, \quad s_{j}^{i} \in B^{*}$,

(6) $\lambda_{j}^{i} \geq 0, \quad s_{j}^{i} \notin B^{*}$,

(7) $\quad t \geq 0$,

(8) $-t+1 \geq 0$.

Theorem 3.1 implies that $(t, \sigma) \in \mathcal{L}\left(\Gamma, p, B^{*}\right)$ if and only if there exists $\lambda \in \mathbb{R}^{m^{*}}$ and $\mu \in \mathbb{R}^{n}$ such that the equalities (1)-(4) and the inequalities (5)-(8) are satisfied.

Theorem 3.1 Let a game $\Gamma \in \mathcal{G}$ and a prior $p \in \Sigma$ be given. For all admissible subsets $B^{*}$ of $S^{*}$, the sets $\mathcal{L}\left(\Gamma, p, B^{*}\right)$ and $\mathcal{O}\left(\Gamma, p, B^{*}\right)$ are $C^{\infty}$ diffeomorphic.

Proof Let $B^{*}$ be an admissible subset of $S^{*}$. For every $i \in N$ we take an element $s_{j}^{i} \in B^{*}$. We define a function $f:[0,1] \times \Sigma \rightarrow \mathbb{R} \times \mathbb{R}^{m^{*}} \times \mathbb{R}^{m^{*}} \times \mathbb{R}^{n}$ by $f(t, \sigma)=(t, \sigma, \lambda, \mu)$, where 
$\lambda_{\ell}^{i}=v^{i}\left(t, \sigma^{-i}, s_{j}^{i}\right)-v^{i}\left(t, \sigma^{-i}, s_{\ell}^{i}\right)$ and $\mu^{i}=v^{i}\left(t, \sigma^{-i}, s_{j}^{i}\right)$. Then $f(t, \sigma) \in \mathcal{O}\left(\Gamma, p, B^{*}\right)$ if and only if $(t, \sigma) \in \mathcal{L}\left(\Gamma, p, B^{*}\right)$. Note that $f$ defined in this way is a $C^{\infty}$ diffeomorphism.

From Theorem 3.1 it follows that for all $(t, \sigma) \in \mathcal{L}\left(\Gamma, p, B^{*}\right)$ there is a unique $\lambda$ and a unique $\mu$ such that $(t, \sigma, \lambda, \mu) \in \mathcal{O}\left(\Gamma, p, B^{*}\right)$. Vice versa, for all $(t, \sigma, \lambda, \mu) \in \mathcal{O}\left(\Gamma, p, B^{*}\right)$ it holds that $(t, \sigma) \in \mathcal{L}\left(\Gamma, p, B^{*}\right)$.

The analysis of the system of equalities and inequalities (1)-(8) provides the following result.

Theorem 3.2 For an open set of games $\Gamma \in \mathcal{G}$ and priors $p \in \Sigma$ with full Lebesgue measure, ${ }^{1}$ for all admissible subsets $B^{*}$ of $S^{*}, \mathcal{L}\left(\Gamma, p, B^{*}\right)$ is a compact 1-dimensional $C^{\infty}$ manifold with boundary. Moreover, $(t, \sigma)$ is a boundary point of $\mathcal{L}\left(\Gamma, p, B^{*}\right)$ if and only if either $\sigma_{j}^{i}=0$ for exactly one $s_{j}^{i} \in B^{*}$, or exactly one $s_{j}^{i} \notin B^{*}$ is a best response to $\sigma^{-i}$, or $t=0$, or $t=1$.

Proof See Appendix.

This theorem implies that, for almost every $\Gamma$ and $p$, for all admissible sets $B^{*}$ of $S^{*}$ the set $\mathcal{L}\left(\Gamma, p, B^{*}\right)$ consists of a finite number of smooth arcs and loops. ${ }^{2}$ The structure of $\mathcal{L}\left(\Gamma, p, B^{*}\right)$ is therefore a simple one; all kinds of complications like bifurcations, spirals, higher dimensional solution sets, diverging behavior, etc. are excluded. Theorem 3.2 does not claim that the set $\mathcal{L}\left(\Gamma, p, B^{*}\right)$ is non-empty. Since the empty set qualifies as a $k$-dimensional manifold for any $k$ by definition, a 1-dimensional manifold may be empty.

To prove Theorem 3.2, it is first shown that, for generic $\Gamma$ and $p$, the set $\mathcal{O}\left(\Gamma, p, B^{*}\right)$ is a compact 1-dimensional $C^{\infty}$ manifold with boundary. By Theorem 3.1, this property carries over to $\mathcal{L}\left(\Gamma, p, B^{*}\right)$. Notice that the set $\mathcal{O}\left(\Gamma, p, B^{*}\right)$ is described in (1)-(8) as a system of equalities and inequalities with $2 m^{*}+n$ equations and $2 m^{*}+n+1$ unknowns. A 1-dimensional solution set is therefore what one expects.

The set $\mathcal{L}\left(\Gamma, p, B^{*}\right)$ consists of a finite number of arcs and loops. If it contains some arcs, it also has a boundary. The next result makes some very precise statements on the properties of boundary points.

Theorem 3.3 For an open set of games $\Gamma \in \mathcal{G}$ and priors $p \in \Sigma$ with full Lebesgue measure, a boundary point $(t, \sigma)$ of $\mathcal{L}\left(\Gamma, p, B^{*}\right)$ is either

\footnotetext{
${ }^{1}$ The set $\mathcal{G}$ is endowed with a measure in the standard way, for details see for instance van Damme (1987), page 25.

${ }^{2}$ Let $X$ be a topological space. It is called an arc if it is homeomorphic to the closed unit interval $[0,1]$ and it has two boundary points in this case; it is called a loop if it is homeomorphic to the unit circle in $\mathbb{R}^{2}$ and it has no boundary points in that case.
} 
(i) not a boundary point of $\mathcal{L}\left(\Gamma, p, \bar{B}^{*}\right)$ for all $\bar{B}^{*} \neq B^{*}$ and lies in $\{0,1\} \times \Sigma$, or

(ii) is a boundary point of exactly one $\mathcal{L}\left(\Gamma, p, \bar{B}^{*}\right)$ with $\bar{B}^{*} \neq B^{*}$ and belongs to $(0,1) \times \Sigma$. Moreover, $B^{*}$ and $\bar{B}^{*}$ differ in exactly one element, say $s_{j}^{i}$, for which $\sigma_{j}^{i}=0$ and $s_{j}^{i}$ is a best response to $\sigma^{-i}$ in $\Gamma^{t}$.

Proof By Theorem 3.2 it follows that in a boundary point of $\mathcal{L}\left(\Gamma, p, B^{*}\right)$ exactly one of the inequalities (5)-(8) is binding. If the binding inequality is (7) or (8) alternative (i) holds. If one of the inequalities from (5) or (6) is binding, the second alternative holds. More precisely, if an inequality from (5) is binding, it holds for exactly one $s_{j}^{i} \in B^{*}$ that $\sigma_{j}^{i}=0$ and the boundary point of $\mathcal{L}\left(\Gamma, p, B^{*}\right)$ is also a boundary point of $\mathcal{L}\left(\Gamma, p, \bar{B}^{*}\right)$, where $\bar{B}^{*}=B^{*} \backslash\left\{s_{j}^{i}\right\}$; if an inequality from (6) is binding, it holds that $\lambda_{j}^{i}=0$ for exactly one $s_{j}^{i} \notin B^{*}$ and the boundary point of $\mathcal{L}\left(\Gamma, p, B^{*}\right)$ is also a boundary point of $\mathcal{L}\left(\Gamma, p, \bar{B}^{*}\right)$, where $\bar{B}^{*}=B^{*} \cup\left\{s_{j}^{i}\right\}$.

If $\mathcal{L}\left(\Gamma, p, B^{*}\right)$ has a boundary point in $(0,1) \times \Sigma$, then there is a unique admissible subset $\bar{B}^{*}$ such that $\mathcal{L}\left(\Gamma, p, \bar{B}^{*}\right)$ has this boundary point in common with $\mathcal{L}\left(\Gamma, p, B^{*}\right)$. The cardinality of $\bar{B}^{*}$ is one less or one greater than the cardinality of $B^{*}$, depending on whether in the common boundary point a strategy in $B^{*}$ is played with probability zero, or whether a strategy not in $B^{*}$ is a best response. The uniqueness of the set $\bar{B}^{*}$ implies that the sets $\mathcal{L}\left(\Gamma, p, B^{*}\right)$ and $\mathcal{L}\left(\Gamma, p, \bar{B}^{*}\right)$ are nicely linked to each other. Generically, it does not happen that three sets have a common boundary point, nor does it happen that a boundary point of one set intersects the interior of another set. All this implies that $\mathcal{L}(\Gamma, p)$ does not display bifurcations.

If $\mathcal{L}\left(\Gamma, p, B^{*}\right)$ has a boundary point in $\{0,1\} \times \Sigma$, then this point does not belong to any other set $\mathcal{L}\left(\Gamma, p, B^{*}\right)$. This implies that such a boundary point is also a boundary point of $\mathcal{L}(\Gamma, p)$.

Formally, the structure of $\mathcal{L}(\Gamma, p)$ is as follows.

Theorem 3.4 For an open set of games $\Gamma \in \mathcal{G}$ and priors $p \in \Sigma$ with full Lebesgue measure, $\mathcal{L}(\Gamma, p)$ is a compact 1-dimensional piecewise $C^{\infty}$ manifold with boundary. ${ }^{3}$ The boundary of $\mathcal{L}(\Gamma, p)$ is given by the intersection of $\mathcal{L}(\Gamma, p)$ and $\{0,1\} \times \Sigma$. There is a unique boundary point in $\{0\} \times \Sigma$.

Proof See Appendix.

For almost every $\Gamma$ and $p$, the set $\mathcal{L}(\Gamma, p)$ consists of a finite number of arcs and loops.

\footnotetext{
${ }^{3} \mathrm{~A}$ compact 1-dimensional piecewise $C^{\infty}$ manifold with boundary is a finite collection of arcs and loops with at most a finite number of non-differentiabilities.
} 
Although it is not necessarily the case that these arcs and loops are smooth, the number of non-differentiabilities is finite at most. Theorem 3.3 implies that all $\operatorname{arcs}$ in $\mathcal{L}(\Gamma, p)$ start and end in $\{0,1\} \times \Sigma$. Each such path consists of a finite sequence of smooth arcs of the sets $\mathcal{L}\left(\Gamma, p, B^{*}\right)$. A loop in $\mathcal{L}(\Gamma, p)$ consists either of a finite sequence (at least two) of differentiable arcs of the sets $\mathcal{L}\left(\Gamma, p, B^{*}\right)$ or is a loop of the set $\mathcal{L}\left(\Gamma, p, B^{*}\right)$.

Generically, each player $i$ has a unique best response to the prior, so generically there is exactly one point of $\mathcal{L}(\Gamma, p)$ that belongs to $\{0\} \times \Sigma$. This point is both a corner point of $\{0\} \times \Sigma$ and a boundary point of $\mathcal{L}\left(\Gamma, p, B^{*}\right)$, where $B^{*}$ consists of the following $n$ strategies: for each player the best response to the prior. By Theorem 3.4 this point is the starting point of a uniquely defined $\operatorname{arc}$ of $\mathcal{L}(\Gamma, p)$. This arc is the the unique feasible path of $\mathcal{L}(\Gamma, p)$ that transforms prior beliefs into equilibrium beliefs.

Corollary 3.5 For an open set of games $\Gamma \in \mathcal{G}$ and priors $p \in \Sigma$ with full Lebesgue measure, the linear tracing procedure is well-defined.

By following the feasible path starting in the unique point $\mathcal{L}(\Gamma, p) \cap(\{0\} \cap \Sigma)$ we find a Nash equilibrium of the game $\Gamma$. The set $\mathcal{L}(\Gamma, p) \cap(\{1\} \times \Sigma)$ consists of all equilibria of the game $\Gamma$. Precisely one of these equilibria is an element of the feasible path of $\mathcal{L}(\Gamma, p)$. Any other equilibrium is a boundary point of $\mathcal{L}(\Gamma, p)$ and is therefore part of some arc of $\mathcal{L}(\Gamma, p)$. A moment of reflection makes clear that the remaining equilibria are pairwise connected by arcs from $\mathcal{L}(\Gamma, p)$, and so the number of Nash equilibria is odd.

Corollary 3.6 For an open set of games $\Gamma \in \mathcal{G}$ with full Lebesgue measure, the number of Nash equilibria is odd.

We illustrate the theorems by means of an example. Consider the game $\Gamma$ depicted in Figure 1 coming from Harsanyi and Selten (1988). We take for the prior $p=\left(p^{1}, p^{2}\right)=$ $\left(\left(\frac{1}{2}, \frac{1}{2}\right),\left(\frac{2}{3}, \frac{1}{3}\right)\right)$. This prior yields the game $\Gamma^{0}$ depicted in Figure 2 as our starting game.

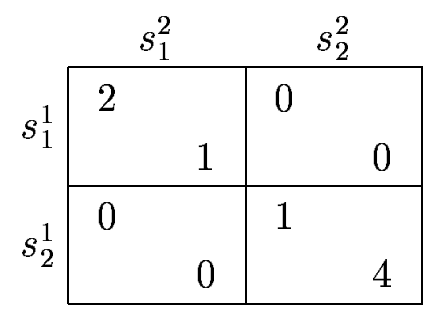

Figure 1: Game $\Gamma$.

In Table 1 the set $\mathcal{L}\left(\Gamma, p, B^{*}\right)$ is described for all admissible sets $B^{*}$. Figure 3 shows the entire graph of the linear tracing procedure $\mathcal{L}(\Gamma, p)=\bigcup_{B^{*}} \mathcal{L}\left(\Gamma, p, B^{*}\right)$. The feasible 

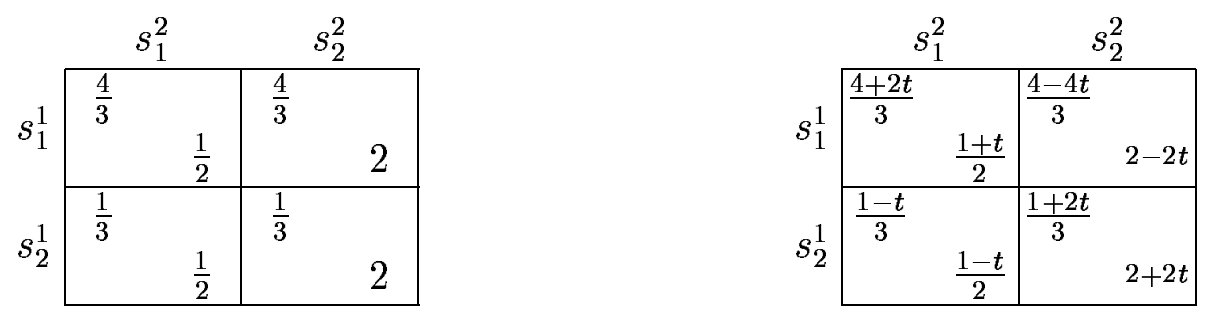

Figure 2: Games $\Gamma^{0}$ and $\Gamma^{t}$.

\begin{tabular}{|l|l|}
\hline \multicolumn{1}{|c|}{$B^{*}$} & $\mathcal{L}\left(\Gamma, p, B^{*}\right)$ \\
\hline$\left\{s_{1}^{1}, s_{1}^{2}\right\}$ & $\left\{\left(t,\left(s_{1}^{1}, s_{1}^{2}\right)\right) \mid t \in\left[\frac{3}{5}, 1\right]\right\}$ \\
$\left\{s_{1}^{1}, s_{2}^{2}\right\}$ & $\left\{\left(t,\left(s_{1}^{1}, s_{2}^{2}\right)\right) \mid t \in\left[0, \frac{1}{2}\right]\right\}$ \\
$\left\{s_{2}^{1}, s_{1}^{2}\right\}$ & $\emptyset$ \\
$\left\{s_{2}^{1}, s_{2}^{2}\right\}$ & $\left\{\left(t,\left(s_{2}^{1}, s_{2}^{2}\right)\right) \mid t \in\left[\frac{1}{2}, 1\right]\right\}$ \\
$\left\{s_{1}^{1}, s_{1}^{2}, s_{2}^{2}\right\}$ & $\left\{\left(\frac{3}{5},\left(s_{1}^{1},(r, 1-r)\right)\right) \mid r \in\left[\frac{1}{9}, 1\right]\right\}$ \\
$\left\{s_{2}^{1}, s_{1}^{2}, s_{2}^{2}\right\}$ & $\emptyset$ \\
$\left\{s_{1}^{1}, s_{2}^{1}, s_{1}^{2}\right\}$ & $\emptyset$ \\
$\left\{s_{1}^{1}, s_{2}^{1}, s_{2}^{2}\right\}$ & $\left\{\left(\frac{1}{2},\left((r, 1-r), s_{2}^{2}\right)\right) \mid r \in[0,1]\right\}$ \\
$\left\{s_{1}^{1}, s_{2}^{1}, s_{1}^{2}, s_{2}^{2}\right\}$ & $\left\{\left(t,\left(\left(\frac{5 t+3}{10 t}, \frac{5 t-3}{10 t}\right),\left(\frac{2 t-1}{3 t}, \frac{t+1}{3 t}\right)\right)\right) \mid t \in\left[\frac{3}{5}, 1\right]\right\}$ \\
\hline
\end{tabular}

Table 1: Description of the sets $\mathcal{L}\left(\Gamma, p, B^{*}\right)$.

path of the linear tracing procedure starts in the point $\left(0,\left(s_{1}^{1}, s_{2}^{2}\right)\right)$ with $B^{*}=\left\{s_{1}^{1}, s_{2}^{2}\right\}$. As long as $t<\frac{1}{2}$, the equalities (1)-(4) determine the feasible path. At $t=\frac{1}{2}$ inequality (6) belonging to strategy $s_{2}^{1}$ is binding, which implies that the second pure strategy of player 1 is a best response to a belief of player 1 that puts probability $1 / 2$ on the prior and probability $1 / 2$ on strategy $s_{2}^{2}$. A further continuation of the path determined by the equalities (1)-(4) without changing the set $B^{*}$ would make the value of $\lambda_{2}^{1}$ negative and thereby violates inequality (6). We have to expand $B^{*}$ by including strategy $s_{2}^{1}$. Our new $B^{*}$ becomes $\left\{s_{1}^{1}, s_{2}^{1}, s_{2}^{2}\right\}$. When we continue following the feasible path from $\left(\frac{1}{2},\left(s_{1}^{1}, s_{2}^{2}\right)\right)$, we reach the point $\left(\frac{1}{2},\left(s_{2}^{1}, s_{2}^{2}\right)\right)$. Continuing with the current $B^{*}$ violates inequality (5), since $\sigma_{1}^{1}$ becomes negative. We delete strategy $s_{1}^{1}$ from $B^{*}$ and our new $B^{*}$ becomes $\left\{s_{2}^{1}, s_{2}^{2}\right\}$. The equalities (1)-(4) related to this $B^{*}$ determine the remainder of the path leading to a Nash equilibrium of the game.

The observations made so far suggest the following algorithm for the computation of the Nash equilibrium selected by the tracing procedure in $n$-person games. Define 


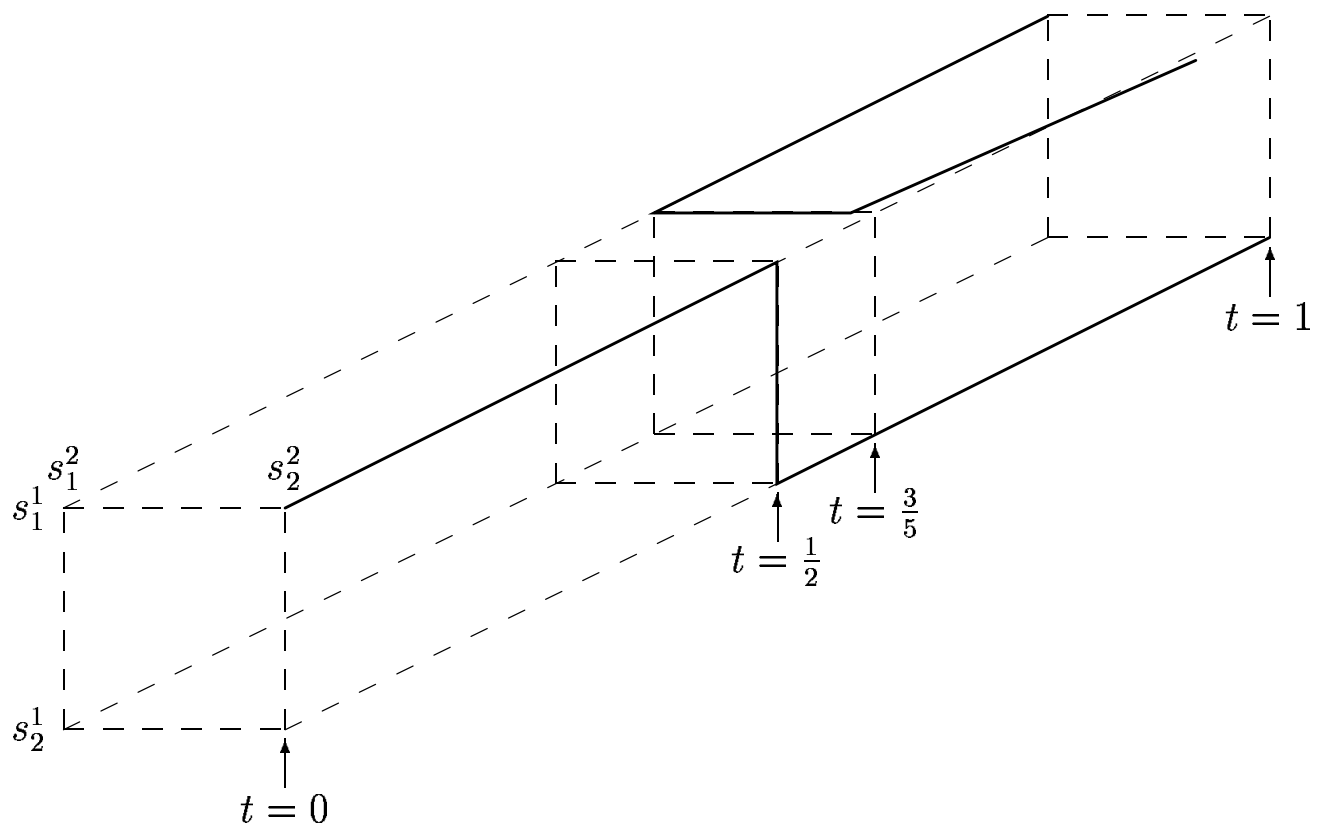

Figure 3: The linear tracing procedure.

the admissible set $B^{*}$ that contains for each player $i$ the best response to the prior and start with a point $(0, \sigma)$ in $\mathcal{L}\left(\Gamma, p, B^{*}\right)$ such that $\sigma^{i}$ is a best response of player $i$ to the prior. Theorem 3.4 implies that $B^{*}$ and $\sigma$ are uniquely determined. The equalities (1)(4) belonging to this $B^{*}$ determine at least a part of the feasible path. As long as the inequalities (5)-(8) hold with strict inequality we do not have to change our $B^{*}$. As soon as one of the inequalities from (5) or (6) gets binding, we have to change the set $B^{*}$. When the binding inequality belongs to (5), say $\sigma_{j}^{i}=0$ while $s_{j}^{i} \in B^{*}$, we have to delete $s_{j}^{i}$ out of $B^{*}$. Obviously, this cannot happen for the starting $B^{*}$. If the binding inequality belongs to (6), say $\lambda_{j}^{i}=0$ while $s_{j}^{i} \notin B^{*}$, we have to add strategy $s_{j}^{i}$ to $B^{*}$. In both situations there is a strategy $s_{j}^{i}$ for which $\sigma_{j}^{i}=0$ and $\lambda_{j}^{i}=0$. In general this leads to a kink in the feasible path of the linear tracing procedure. We repeat this procedure with our new $B^{*}$. Note that inequality (7) is only binding in the starting point. When inequality (8) is binding, a Nash equilibrium is found.

In Figure 3 we have seen that the linear tracing procedure determined a whole range of equilibria at $t=\frac{1}{2}$. In general, it is even possible that the linear tracing procedure buckles and moves backwards in $t$ (see Garcia and Zangwill (1981) and section 4.19 of Harsanyi and Selten (1988)). 


\section{A Differentiable Approach}

The previous section presents a method that can be used for the computation of a Nash equilibrium, in particular the Nash equilibrium selected by the Harsanyi-Selten theory. A potential drawback of that method is that one has to check out all the time whether the system of equalities used is still appropriate, and if not, one has to switch between different systems of equalities.

The idea of switching homotopies has only been used in the literature recently. In the context of general equilibrium theory with incomplete markets switching homotopies are used by Brown, DeMarzo and Eaves (1996). In our set-up, switching homotopies can be a serious problem in terms of computing time. There are $\prod_{i \in N}\left(2^{m^{i}}-1\right)$ different sets $B^{*}$, whereas each one of them may be generated several times in the course of the algorithm.

We show that for the problem of computing Nash equilibria, switching homotopies can be avoided. It is possible to formulate one, everywhere differentiable, homotopy. To obtain differentiability a well-chosen transformation of variables is used, which is also used in Garcia and Zangwill (1981), and Herings and Schmedders (1999). Define for all $\alpha \in \mathbb{R}^{m^{*}}$

$$
\sigma_{j}^{i}(\alpha)=\left[\max \left\{0, \alpha_{j}^{i}\right\}\right]^{2} \quad \text { and } \quad \lambda_{j}^{i}(\alpha)=\left[\max \left\{0,-\alpha_{j}^{i}\right\}\right]^{2}
$$

After this transformation of variables, the Kuhn-Tucker first-order conditions that provide necessary and sufficient conditions for $\sigma^{i}$ to be a best response against $\bar{\sigma}^{-i}$ in the game $\Gamma^{\bar{t}}$ become

$$
\begin{aligned}
& v^{i}\left(\bar{t}, \bar{\sigma}^{-i}(\alpha), s_{j}^{i}\right)+\lambda_{j}^{i}(\alpha)-\mu^{i}=0, \quad s_{j}^{i} \in S^{*}, \\
& \lambda_{j}^{i}(\alpha) \geq 0, \quad \sigma_{j}^{i}(\alpha) \geq 0, \quad \lambda_{j}^{i}(\alpha) \sigma_{j}^{i}(\alpha)=0, \quad s_{j}^{i} \in S^{*} \\
& \sum_{j=1}^{m^{i}} \sigma_{j}^{i}(\alpha)-1=0, \quad i \in N .
\end{aligned}
$$

Owing to the proposed transformation of variables, the conditions $\lambda_{j}^{i}(\alpha) \geq 0, \sigma_{j}^{i}(\alpha) \geq 0$ and $\lambda_{j}^{i}(\alpha) \sigma_{j}^{i}(\alpha)=0$ are trivially satisfied. We can reformulate the (in)equalities in (1)-(8) that characterize the set $\mathcal{O}\left(\Gamma, p, B^{*}\right)$ by considering solutions $(t, \alpha, \mu) \in \mathbb{R} \times \mathbb{R}^{m^{*}} \times \mathbb{R}^{n}$ with $\alpha_{j}^{i} \geq 0$ if $s_{j}^{i} \in B^{*}$ and $\alpha_{j}^{i} \leq 0$ if $s_{j}^{i} \notin B^{*}$ such that

(a) $v^{i}\left(t, \sigma^{-i}(\alpha), s_{j}^{i}\right)+\lambda_{j}^{i}(\alpha)-\mu^{i}=0, \quad s_{j}^{i} \in S^{*}$,

(b) $\quad \sum_{j=1}^{m^{i}} \sigma_{j}^{i}(\alpha)-1=0, \quad i \in N$,

(c) $\quad t \geq 0$,

(d) $\quad-t+1 \geq 0$.

Considering the system of equalities and inequalities (a)-(d) it is striking that the set $B^{*}$ has disappeared. The same system of equalities and inequalities can be used, irrespective 
of the set $B^{*}$. The role of $B^{*}$ is taken over by the vector $\alpha$; more precisely, by the signcombinations of the components of $\alpha$. Figure 4 illustrates the relation between the vector $\alpha$ and the set $B^{*}$.
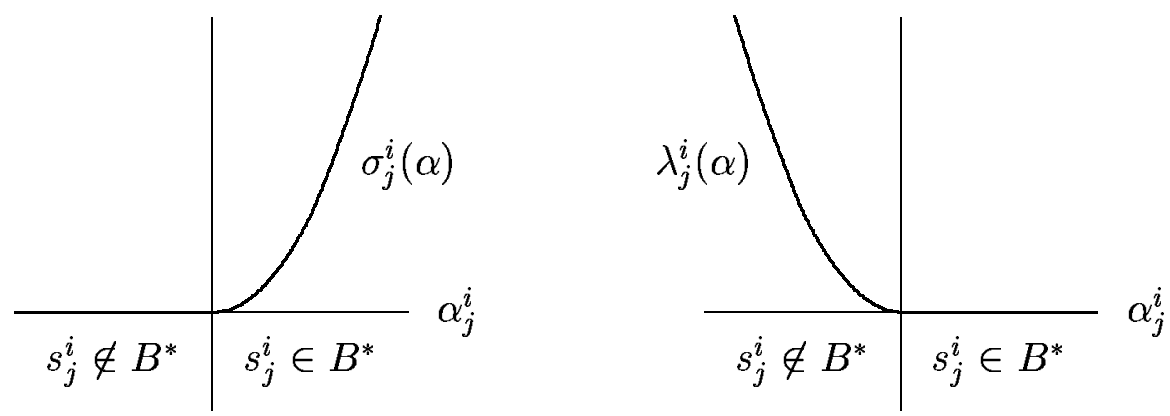

Figure 4: Relation between $\sigma_{j}^{i}$ and $\alpha_{j}^{i}$ and between $\lambda_{j}^{i}$ and $\alpha_{j}^{i}$.

Counting equations and unknowns in the system (a)-(d) shows that there is one degree of freedom, and therefore one expects a 1-dimensional solution set. Consider any solution $(t, \alpha, \mu)$ to (a)-(d). When $\alpha_{j}^{i}=0$, then both $\sigma_{j}^{i}(\alpha)$ and $\lambda_{j}^{i}(\alpha)$ are zero. This implies that we have exactly two admissible subsets of $S^{*}$ for which the set of (in)equalities (1)-(8) are satisfied. If along a solution curve $\alpha_{j}^{i}$ is increasing while passing zero, then $\sigma_{j}^{i}(\alpha)$ gets positive and $B_{\text {new }}^{*}=B_{\text {old }}^{*} \cup\left\{s_{j}^{i}\right\}$. If $\alpha_{j}^{i}$ is decreasing while passing zero, then $\lambda_{j}^{i}(\alpha)$ gets negative and $B_{\text {new }}^{*}=B_{\text {old }}^{*} \backslash\left\{s_{j}^{i}\right\}$. When $\alpha_{j}^{i}$ passes zero, a kink appears in the method proposed in Section 3.

The left-hand sides of the equalities (a)-(b) specify the homotopy $\mathcal{H}:[0,1] \times \mathbb{R}^{m^{*}} \times \mathbb{R}^{n} \rightarrow$ $\mathbb{R}^{m^{*}} \times \mathbb{R}^{n}$

$$
\mathcal{H}(t, \alpha, \mu)=\left(\begin{array}{c}
v^{i}\left(t, \sigma^{-i}(\alpha), s_{j}^{i}\right)+\lambda_{j}^{i}(\alpha)-\mu^{i}, \quad s_{j}^{i} \in S^{*} \\
\sum_{j=1}^{m^{i}} \sigma_{j}^{i}(\alpha)-1, \quad i \in N
\end{array}\right) .
$$

The homotopy function $\mathcal{H}$ is continuously differentiable. The inequalities (c) and (d) are satisfied as the homotopy takes $[0,1]$ as the domain for the variable $t$. It has the salient feature that its zeros desribe the linear tracing procedure, $(t, \alpha, \mu) \in \mathcal{H}^{-1}(\{0\}) \Leftrightarrow$ $(t, \sigma(\alpha)) \in \mathcal{L}(\Gamma, p)$. Starting at the unique point $\left(0, \alpha^{0}, \mu^{0}\right) \in \mathcal{H}^{-1}(\{0\})$ at $t=0$ and following the path described by the zeros of $\mathcal{H}$, we end up in a point $(1, \tilde{\alpha}, \tilde{\mu}) \in \mathcal{H}^{-1}(\{0\})$. This point generates the Nash equilibrium $\sigma(\tilde{\alpha})$ of $\Gamma$ selected by the Harsanyi-Selten theory.

Theorem 4.1 specifies the structure of the set of solutions to the homotopy, $\mathcal{H}^{-1}(\{0\})$.

Theorem 4.1 For an open set of games $\Gamma \in \mathcal{G}$ and priors $p \in \Sigma$ with full Lebesgue measure, $\mathcal{H}^{-1}(\{0\})$ is a compact 1-dimensional $C^{1}$ manifold with boundary. The boundary 
of $\mathcal{H}^{-1}(\{0\})$ equals the intersection of $\mathcal{H}^{-1}(\{0\})$ and $\{0,1\} \times \mathbb{R}^{m^{*}} \times \mathbb{R}^{n}$. There is a unique boundary point in $\{0\} \times \mathbb{R}^{m^{*}} \times \mathbb{R}^{n}$.

Proof See Appendix.

The set $\mathcal{H}^{-1}(\{0\})$ consists of finitely many differentiable arcs and loops. All arcs start and end in $\{0,1\} \times \mathbb{R}^{m^{*}} \times \mathbb{R}^{n}$. Loops have no points in common with $\{0,1\} \times \mathbb{R}^{m^{*}} \times \mathbb{R}^{n}$. There is exactly one arc that starts in $\{0\} \times \mathbb{R}^{m^{*}} \times \mathbb{R}^{n}$ and that ends in $\{1\} \times \mathbb{R}^{m^{*}} \times \mathbb{R}^{n}$ with a point $(1, \tilde{\alpha}, \tilde{\mu})$ that generates the Nash equilibrium selected by the tracing procedure. This arc is a transformation of the feasible path of the tracing procedure. All other arcs start and end in $\{1\} \times \mathbb{R}^{m^{*}} \times \mathbb{R}^{n}$ and connect two points inducing Nash equilibria of $\Gamma$.

The structure of $\mathcal{H}^{-1}(\{0\})$ is even simpler than the one of $\mathcal{L}(\Gamma, p)$. Not only are complications like bifurcations, spirals, higher dimensional solutions sets, diverging behavior, etc., excluded. The arcs and loops in $\mathcal{H}^{-1}(\{0\})$ are differentiable everywhere. It is the transformation of variables that smooths out the kinks. As a direct consequence, it is possible to calculate the derivate at each point of the feasible path, which makes it possible to follow the path by means of differentiable as opposed to simplicial methods.

We return to the example of the previous section. It is very convenient to describe the variables $t, \sigma, \lambda, \alpha$ and $\mu$ as functions of pathlength $\tau$. If we do so, the feasible path of the linear tracing procedure can be shown by Figure 5.
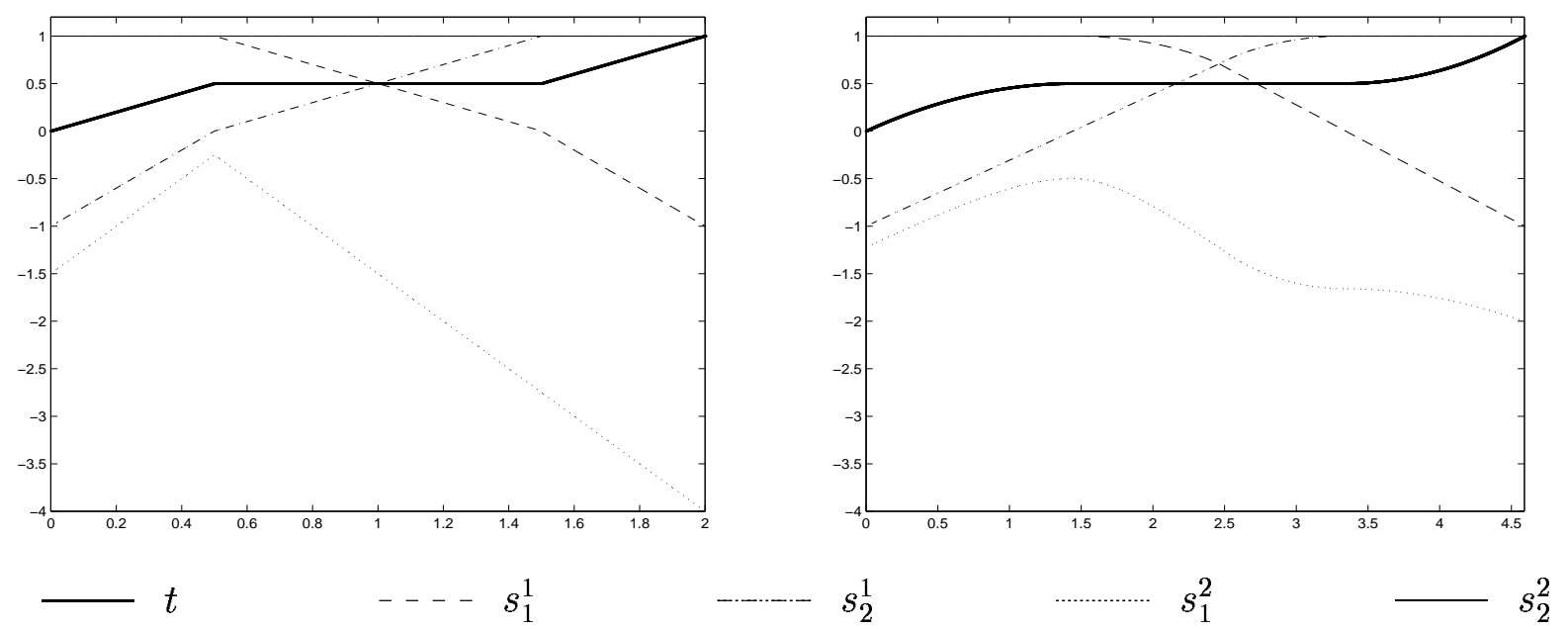

Figure 5: Before and after transformation.

The first plot in Figure 5 shows $t, \sigma$ and $\lambda$ as a function of pathlength. To make the picture more clear, only informative values of the variables are depicted. In particular this means that for each pure strategy $s_{j}^{i}$, either $\sigma_{j}^{i}$ or $-\lambda_{j}^{i}$ is plotted, depending on which one 
is non-zero. The second plot shows the values of $t$ and $\alpha$ as a function of pathlength and therefore corresponds to the feasible path of the tracing procedure after the transformation.

The first plot shows that kinks appear for all variables when $s_{1}^{1}$ and $s_{2}^{1}$ are passing zero. In the second plot no kinks appear at all. In that plot, when $s_{1}^{1}$ or $s_{2}^{1}$ passes zero, the derivatives of all variables with respect to pathlength are equal to zero, except the variable passing zero. This is a general phenomenon and follows from the proofs in the appendix.

\section{Implementation}

This section is devoted to the implementation of the homotopy function. Because of the homotopy path, it is possible to apply standard numerical path-following techniques that are available in professionally programmed software.

We have programmed a number of FORTRAN-subroutines belonging to the softwarepackage HoMPACK ${ }^{4}$, a ForTran77 program (see Watson, Billups and Morgan (1987)).

HOMPACK provides three qualitative different algorithms for tracking the zero curve of the homotopy: ordinary differential equation-based, normal flow, and augmented Jacobian matrix. Separate routines are also provided for dense ans sparse matrices. The algorithm we used for implication is FIXPDF, which is an ordinary differential equation-based algorithm working with dense Jacobian matrices. We used FIXPDF because this algorithm is more robust than the others even though this may come at the cost of higher computing times.

We parameterize the homotopy path by pathlength $\tau$. Thus $t=t(\tau), \alpha=\alpha(\tau)$ and $\mu=\mu(\tau)$ along the homotopy path, and $\mathcal{H}(t(\tau), \alpha(\tau), \mu(\tau))=0$ identically in $\tau$. The differential equation is characterized by

$$
\begin{aligned}
& \frac{\mathrm{d}}{\mathrm{d} \tau} \mathcal{H}(t(\tau), \alpha(\tau), \mu(\tau))=\partial \mathcal{H}(t(\tau), \alpha(\tau), \mu(\tau)) \cdot\left(\begin{array}{c}
\mathrm{d} t / \mathrm{d} \tau \\
\mathrm{d} \alpha / \mathrm{d} \tau \\
\mathrm{d} \mu / \mathrm{d} \tau
\end{array}\right)=0, \\
& \left\|\left(\frac{\mathrm{d} t}{\mathrm{~d} \tau}, \frac{\mathrm{d} \alpha}{\mathrm{d} \tau}, \frac{\mathrm{d} \mu}{\mathrm{d} \tau}\right)\right\|_{2}=1 .
\end{aligned}
$$

and initial conditions are given by

$$
(t(0), \alpha(0), \mu(0))=\mathcal{H}^{-1}(\{0\}) \cap\left(\{0\} \times \mathbb{R}^{m^{*}} \times \mathbb{R}^{n}\right) .
$$

The homotopy path corresponds to the trajectory of the initial value problem. When $t(\bar{\tau})=1,(\alpha(\bar{\tau}), \mu(\bar{\tau}))$ is a zeropoint of $\mathcal{H}$ and $\sigma(\alpha(\bar{\tau}))$ is a Nash equilibrium of game $\Gamma$

\footnotetext{
${ }^{4}$ http://www-fp.mcs.anl.gov/otc/Guide/SoftwareGuide/Blurbs/hompack.html
} 
with equilibrium payoff $\mu(\bar{\tau})$. Complete details for solving the initial value problem are in Watson (1979) and Watson and Fenner (1980).

We have implemented the homotopy function as described in Section 4. It may be possible to improve on computing times by using a transformation with a power less than 2, or by rescaling utilities and probabilities. Moreover, HOMPACK provides the possibility to adjust a number of parameters, mainly related to the accuracy by which the homotopy path is followed. An extensive digress on the optimal numerical implementation of the homotopy is beyond the scope of the current paper.

Among others we applied our program to the example of Harsanyi and Selten from the previous sections. Figure 6 shows five plots. The first plot corresponds to the homotopyvariabele $t$ and is plotted with respect to pathlength $\tau$. Each of the other four plots correspond to a homotopy-variable $\alpha_{j}^{i}, i=1,2, j=1,2$. These variables are also plotted as a function of pathlength. For all five plots, the dotted lines show the analytical solution path and the solid lines show the numerical solution paths.

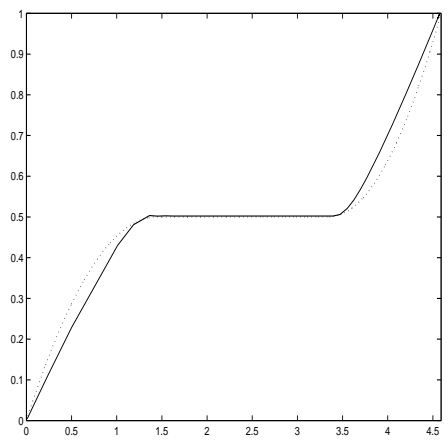

$t$ as a function of $\tau$.

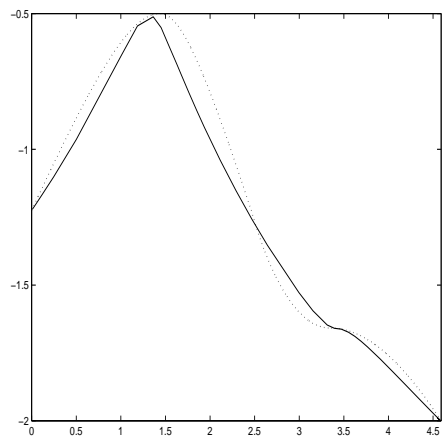

$\alpha_{1}^{2}$ as a function of $\tau$.

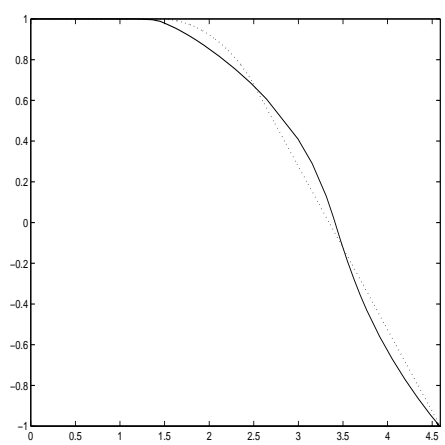

$\alpha_{1}^{1}$ as a function of $\tau$.

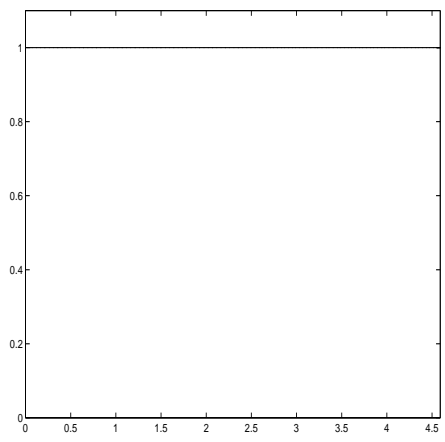

$\alpha_{2}^{2}$ as a function of $\tau$.

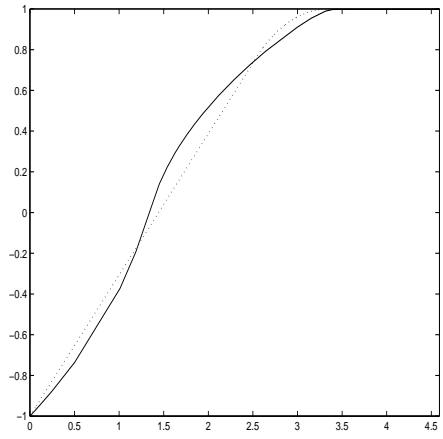

$\alpha_{2}^{1}$ as a function of $\tau$.

Figure 6: The behavior of the homotopy-variables along the zero curve.

For $n=3$ players, each having $m$ strategies, we have generated a randomly chosen game for $m=2, \ldots, 10$. All pay-offs are chosen independently from one another out of 
the uniform distribution on $[0,1]$. The prior is chosen such that the players play all their strategies with equal probability. The following table shows the time (in seconds) and the number of function evaluations ( $\mathrm{NFE}$ ) needed to compute the Nash equilibrium. The maximal inaccuracy of our calculation amounts to $10^{-4}$, which means that the 2-norm of the value of the homotopy function is less than $10^{-4}$ in the computed equilibrium. From the specification of the homotopy function, this implies that the maximal gain in pay-off from a unilateral deviation is equal to $10^{-4}$.

\begin{tabular}{c|ccccccccc}
$m$ & 2 & 3 & 4 & 5 & 6 & 7 & 8 & 9 & 10 \\
\hline time & 0 & 1 & 1 & 6 & 6 & 24 & 37 & 17 & 233 \\
NFE & 43 & 77 & 62 & 194 & 101 & 236 & 183 & 82 & 411
\end{tabular}

Our homotopy also provides the possibility to investigate how sensitive the Nash equilibrium selected by the linear tracing procedure is with respect to the choice of the prior. In a game with $n=4$ players, each having $m=6$ strategies, the total number of Nash equilibria is estimated to be 2.037 , with a standard error of the estimation of 66 . We have fixed one such game and computed the Nash equilibrium for 10 randomly generated priors. We have found 9 different Nash equilibria in this way. This suggests that multiplicity of Nash equilibria is a serious problem in game theory and that the specific method used for equilibrium selection may be of crucial importance.

\section{A Proofs}

To make the proofs as transparent as possible, we need some notations and definitions from the theory of regular constraint sets as presented in Jongen, Jonker and Twilt (1983), see also Herings (1997a) for a first application of this theory in economics.

For some $r \geq 1$ a subset $M$ of $\mathbb{R}^{m}$ is called a $k$-dimensional $C^{r}$ manifold with generalized boundary (MGB), if for every $\bar{x} \in M$ there exists a $C^{r}$ diffeomorphism $\phi: U \rightarrow V$, where $U$ is an open subset of $\mathbb{R}^{m}$ containing $\bar{x}$ and $V$ is open in $\mathbb{R}^{m}$, and some integer $\ell(\bar{x}) \geq 0$, such that $\phi(\bar{x})=0$ and $\phi(U \cap M)$ equals $\left\{y \in V \mid y_{i}=0, i=1, \ldots, m-k\right.$, and $y_{i} \geq 0, i=$ $m-k+1, \ldots, m-k+\ell(\bar{x})\}$. If for every element $\bar{x}$ of an MGB $M$ it holds that $\ell(\bar{x}) \leq 1$, then $M$ is called a manifold with boundary and the set of elements $\bar{x}$ for which $\ell(\bar{x})=1$ is an $(k-1)$-dimensional manifold, called the boundary of $M$.

One way to show that a set is an MGB is by showing that it is a regular contraint set. Let $J^{1}$ and $J^{2}$ be two finite index sets and let $g_{j}$ for all $j \in J^{1}$ and $h_{j}$ for all $j \in J^{2}$, be $C^{r}$ functions defined on some open subset $X$ of $\mathbb{R}^{m}$. We define

$$
M[g, h]=\left\{x \in X \mid g_{j}(x)=0, \forall j \in J^{1}, \text { and } h_{j}(x) \geq 0, \forall j \in J^{2}\right\} .
$$


For $x \in X$ we define the set $J^{0}(x)=\left\{j \in J^{2} \mid h_{j}(x)=0\right\}$. If for every $\bar{x} \in M[g, h]$ it holds that

$$
\left\{\partial_{x} g_{j}(\bar{x}) \mid j \in J^{1}\right\} \cup\left\{\partial_{x} h_{j}(\bar{x}) \mid j \in J^{0}(\bar{x})\right\}
$$

is a set of independent vectors, then $M[g, h]$ is called a $C^{r}$ regular constraint set (RCS). In Jongen, Jonker and Twilt (1983) it is shown that every $C^{r} \mathrm{RCS}$ is an $\left(m-\left|J^{1}\right|\right)$-dimensional $C^{r}$ MGB with $\ell(\bar{x})=\left|J^{0}(\bar{x})\right|$ for every $\bar{x} \in M[g, h]$.

Fix the number of pure strategies for each player, and fix a prior $p \in \Sigma$. For any tuple of utility functions $u$ and any admissible subset $B^{*}$, we define the functions $g^{B^{*}, u}$ : $\mathbb{R}^{1+2 m^{*}+n} \rightarrow \mathbb{R}^{2 m^{*}+n}$ and $h^{B^{*}, u}: \mathbb{R}^{1+2 m^{*}+n} \rightarrow \mathbb{R}^{m^{*}+2}$ in such a way that $g^{B^{*}, u}$ equals the left-hand side of the equalities (1)-(4) and $h^{B^{*}, u}$ the left-hand side of the inequalities (5)-(8),

$$
g^{B^{*}, u}(t, \sigma, \lambda, \mu)=\left(\begin{array}{c}
v^{i}\left(t, \sigma^{-i}, s_{j}^{i}\right)+\lambda_{j}^{i}-\mu^{i}, \quad s_{j}^{i} \in S^{*} \\
\sigma_{j}^{i}, \quad s_{j}^{i} \notin B^{*} \\
\lambda_{j}^{i}, \quad s_{j}^{i} \in B^{*} \\
\sum_{j=1}^{m^{i}} \sigma_{j}^{i}-1, \quad i \in N
\end{array}\right)
$$

and

$$
h^{B^{*}, u}(t, \sigma, \lambda, \mu)=\left(\begin{array}{cc}
\sigma_{j}^{i}, & s_{j}^{i} \in B^{*} \\
\lambda_{j}^{i}, & s_{j}^{i} \notin B^{*} \\
& t \\
-t+1
\end{array}\right)
$$

A point $(t, \sigma, \lambda, \mu) \in \mathcal{O}\left(\Gamma, p, B^{*}\right)$ is a solution of (1)-(8) if and only if $g^{B^{*}, u}(t, \sigma, \lambda, \mu)=0$ and $h^{B^{*}, u}(t, \sigma, \lambda, \mu) \geq 0$.

Once the number of pure strategies of each player is fixed, a noncooperative game is completely determined by the tuple of utility functions $u$, which can be represented by a vector in $\mathbb{R}^{m n}$. The standard topology and measure on $\mathbb{R}^{m n}$ therefore induce a topology and a measure on games. For every $u \in \mathbb{R}^{m n}$, we define the functions $g^{B^{*}}: \mathbb{R}^{1+2 m^{*}+n} \times$ $\mathbb{R}^{n m} \rightarrow \mathbb{R}^{2 m^{*}+n}$ and $h^{B^{*}}: \mathbb{R}^{1+2 m^{*}+n} \times \mathbb{R}^{n m} \rightarrow \mathbb{R}^{m^{*}+2}$ by $g^{B^{*}}(t, \sigma, \lambda, \mu, u)=g^{B^{*}, u}(t, \sigma, \lambda, \mu)$ and $h^{B^{*}}(t, \sigma, \lambda, \mu, u)=h^{B^{*}, u}(t, \sigma, \lambda, \mu)$. Figure 7 presents the matrix of derivatives of the functions $g^{B^{*}}$ and $h^{B^{*}}$ with respect to all variables.

Each row in Figure 7 corresponds to one of the equalities and inequalities in (1)-(8). To make the figure more clear, derivatives with respect to $\sigma$ are first taken for $s_{j}^{i} \in B^{*}$. The first applies to the derivatives with respect to $\lambda$ and the ordering of the equalities in (1). From the properties listed below the matrix, it follows that the matrices $\partial_{u} v$ and $E$ have full row rank. 


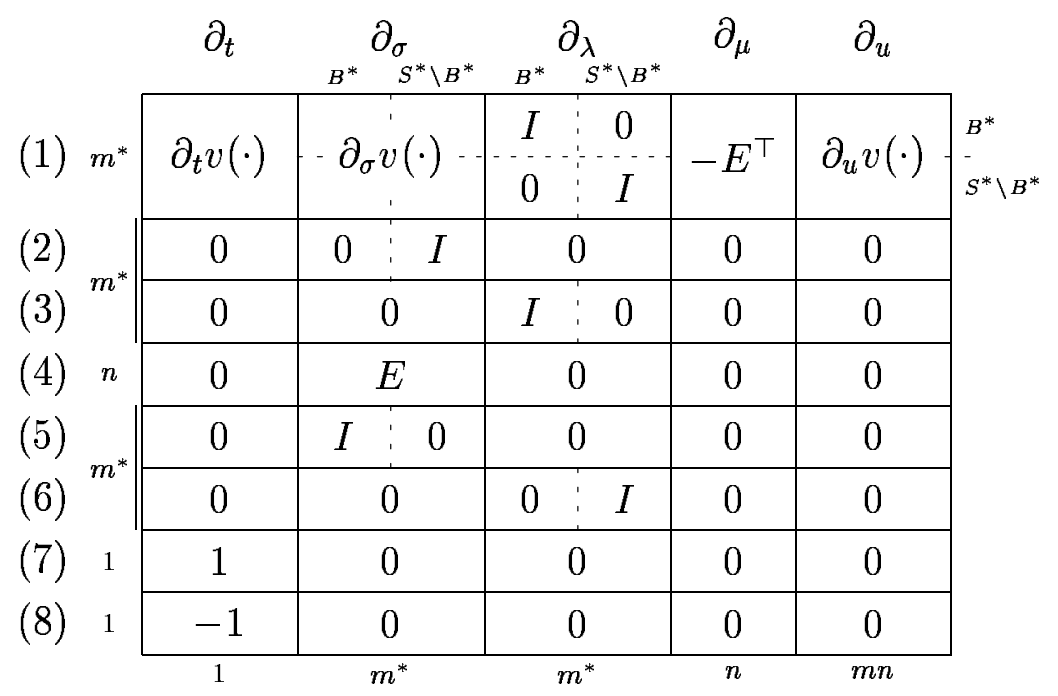

where

$$
\begin{aligned}
& E\left(i, s_{j^{\prime}}^{i^{\prime}}\right)= \begin{cases}1 & \text { if } i=i^{\prime} \\
0 & \text { otherwise }\end{cases} \\
& \partial_{u^{k}\left(s^{-k}, s_{\ell}^{k}\right)} v^{i}\left(t, \sigma^{-i}, s_{j}^{i}\right)=0 \quad \text { if } s_{j}^{i} \neq s_{\ell}^{k} \quad \text { for all } s^{-k} \in S^{-k} \\
& \sum_{s^{-i} \in S^{-i}} \partial_{u^{i}\left(s^{-i}, s_{j}^{i}\right)} v^{i}\left(t, \sigma^{-i}, s_{j}^{i}\right)=1 .
\end{aligned}
$$

Figure 7: The matrix of derivatives of $g^{B^{*}}$ and $h^{B^{*}}$.

The structure of the proof of Theorem 3.2 is as follows. First, it is shown that for almost all games $u, M\left[g^{B^{*}, u}, h^{B^{*}, u}\right]$ is a regular constraint set, from which the manifold structure of $\mathcal{L}\left(\Gamma, p, B^{*}\right)$ follows immediately. Next it is shown that there is an open set of full measure for which the manifold structure holds. The proof of the first part is done when it is shown that for almost all games $u$, for every $\bar{x} \in M\left[g^{B^{*}, u}, h^{B^{*}, u}\right]$,

$$
\left\{\partial_{x} g_{j}^{B^{*}, u}(\bar{x}) \mid j \in J^{1}\right\} \cup\left\{\partial_{x} h_{j}^{B^{*}, u}(\bar{x}) \mid j \in J^{0}(\bar{x})\right\}
$$

is a set of independent vectors. To show this we need three lemmas, Lemmas A.1, A.2 and A.3. Lemma A.1 is used to handle points $\bar{x}$ for which $J^{0}(\bar{x})=\emptyset$, Lemma A.2 deals with points $\bar{x}$ such that the cardinality of $J^{0}(\bar{x})$ is one, and Lemma A.3 implies that $J^{0}(\bar{x})$ contains one element at most.

Lemma A.1 Let a prior $p \in \Sigma$ and an admissible subset $B^{*}$ of $S^{*}$ be given. Then, for almost all $u, g^{B^{*}, u} \pitchfork\{0\}$.

Proof Consider a point $(\bar{t}, \bar{\sigma}, \bar{\lambda}, \bar{\mu}, \bar{u})$ such that $g^{B^{*}}(\bar{t}, \bar{\sigma}, \bar{\lambda}, \bar{\mu}, \bar{u})=0$. The matrix of partial 
derivates of $g^{B^{*}}$ at $(\bar{t}, \bar{\sigma}, \bar{\lambda}, \bar{\mu}, \bar{u})$ is given by the rows (1)-(4) in Figure 7. We show first that this matrix has full row rank, from which it follows that $g^{B^{*}} \pitchfork\{0\}$.

Since $\partial_{u} v(\cdot)$ has full row rank and the derivative with respect to $u$ in (2)-(4) is zero, it is sufficient to show that the part of the matrix given by (2)-(4) has full row rank. Since the derivative with respect to $\lambda$ in (3) has full row rank, whereas the derivative with respect to $\lambda$ in (2) and (4) is zero, it is sufficient to show that the rows in (2) and (4) are independent. The admissibility of $B^{*}$ implies that the derivative with respect to $\sigma_{B^{*}}$ in (4) has full row rank. Since, the derivative with respect to $\sigma_{B^{*}}$ in $(2)$ is zero, the only thing left to prove is that (2) has independent rows, which is obvious from the derivative with respect to $\sigma_{S^{*} \backslash B^{*}}$. Consequently, $g^{B^{*}} \pitchfork\{0\}$.

By the transversality theorem (see, for example Mas-Colell (1985), theorem I.2.2) and since $g^{B^{*}}$ is a $C^{\infty}$ function, it follows that the complement of the set $\left\{u \in \mathbb{R}^{n m} \mid g^{B^{*}, u} \pitchfork\{0\}\right\}$ has Lebesgue measure zero.

Lemma A.2 Let a prior $p \in \Sigma$ and an admissible subset $B^{*}$ of $S^{*}$ be given. Moreover, let an inequality $j^{\prime} \in\left\{1, \ldots, m^{*}+2\right\}$ be given. Then, for almost all $u,\left(g^{B^{*}, u}, h_{j^{\prime}}^{B^{*}, u}\right) \pitchfork\{0\}$.

Proof Consider a point $(\bar{t}, \bar{\sigma}, \bar{\lambda}, \bar{\mu}, \bar{u})$ such that $g^{B^{*}}(\bar{t}, \bar{\sigma}, \bar{\lambda}, \bar{\mu}, \bar{u})=0$ and $h_{j^{\prime}}^{B^{*}}(\bar{t}, \bar{\sigma}, \bar{\lambda}, \bar{\mu}, \bar{u})=$ 0 . The matrix of partial derivates of $\left(g^{B^{*}}, h_{j^{\prime}}^{B^{*}}\right)$ at $(\bar{t}, \bar{\sigma}, \bar{\lambda}, \bar{\mu}, \bar{u})$ is given in Figure 7 by the rows (1)-(4) and a single row related to $h_{j^{\prime}}^{B^{*}}$ in (5)-(8). We show first that this matrix has full row rank, from which it follows that $\left(g^{B^{*}}, h_{j^{\prime}}^{B^{*}}\right) 历\{0\}$.

If row $j^{\prime}$ belongs to $(6),(7)$ or $(8)$, then it follows from the derivative with respect to $\lambda_{S^{*} \backslash B^{*}}$ or from the derivative with respect to $t$ that row $j^{\prime}$ has rank 1 . Since all other derivatives in row $j^{\prime}$ are zero, it follows as in the proof of Lemma A.1 that the rows of (1)-(4) together with row $j^{\prime}$ are independent. Consider the case where row $j^{\prime}$ belongs to (5). Following the first part of the proof of Lemma A.1, it suffices to prove that (2) and (4) together with row $j^{\prime}$ are independent. Inequality $h_{j^{\prime}}^{B^{*}}$ states that $\sigma_{j^{\prime}}^{i} \geq 0, s_{j^{\prime}}^{i} \in B^{*}$, and this inequality is now required to hold with equality. Since $\sum_{j=1}^{m^{i}} \sigma_{j}^{i}-1=0$, it follows that $\left|B^{*} \cap S^{i}\right|>1$. Then the row related to $s_{j^{\prime}}^{i}$ together with (2) and (4) trivially form an independent set. Consequently, $\left(g^{B^{*}}, h_{j^{\prime}}^{B^{*}}\right) 历\{0\}$. By the transversality theorem it follows that the complement of $\left\{u \in \mathbb{R}^{n m} \mid\left(g^{B^{*}, u}, h_{j^{\prime}}^{B^{*}, u}\right) 历\{0\}\right\}$ has Lebesgue measure zero.

Lemma A.3 Let a prior $p \in \Sigma$ and an admissible subset $B^{*}$ of $S^{*}$ be given. Moreover, let inequalities $j^{\prime}, j^{\prime \prime} \in\left\{1, \ldots, m^{*}+2\right\}$ with $j^{\prime} \neq j^{\prime \prime}$ be given. Then, for almost all $u$, $\left(g^{B^{*}, u}, h_{j^{\prime}}^{B^{*}, u}, h_{j^{\prime \prime}}^{B^{*}, u}\right) 历\{0\}$.

Proof Consider a point $(\bar{t}, \bar{\sigma}, \bar{\lambda}, \bar{\mu}, \bar{u})$ such that $g^{B^{*}}(\bar{t}, \bar{\sigma}, \bar{\lambda}, \bar{\mu}, \bar{u})=0, h_{j^{B^{*}}}^{B^{*}}(\bar{t}, \bar{\sigma}, \bar{\lambda}, \bar{\mu}, \bar{u})=0$, and $h_{j^{\prime}}^{B^{*}}(\bar{t}, \bar{\sigma}, \bar{\lambda}, \bar{\mu}, \bar{u})=0$. The matrix of partial derivates of $\left(g^{B^{*}}, h_{j^{\prime}}^{B^{*}}, h_{j^{\prime \prime}}^{B^{*}}\right)$ at $(\bar{t}, \bar{\sigma}, \bar{\lambda}, \bar{\mu}, \bar{u})$ 
is given in Figure 7 by the rows (1)-(4) and two rows related to $h_{j^{\prime}}^{B^{*}}$ and $h_{j^{\prime \prime}}^{B^{*}}$ in (5)-(8). We show first that this matrix has full row rank, from which it follows that $\left(g^{B^{*}}, h_{j^{\prime}}^{B^{*}}, h_{j^{\prime \prime}}^{B^{*}}\right) 历\{0\}$.

The case where the two rows are not equal to (7) and (8) is similar to the proof of Lemma A.2. Rows (7) and (8) are not independent. However, they cannot be binding simultaneously, because then it holds that $t=0$ and $t=1$. Consequently, $\left(g^{B^{*}}, h_{j^{\prime}}^{B^{*}}, h_{j^{\prime \prime}}^{B^{*}}\right) \pitchfork\{0\}$. It follows that the complement of the set $\left\{u \in \mathbb{R}^{n m} \mid\left(g^{B^{*}, u}, h_{j^{\prime}}^{B^{*}, u}, h_{j^{\prime \prime}}^{B^{*}, u}\right) 币\{0\}\right\}$ has Lebesgue measure zero.

Theorem 3.2 For an open set of games $\Gamma \in \mathcal{G}$ and priors $p \in \Sigma$ with full Lebesgue measure, for all admissible subsets $B^{*}$ of $S^{*}, \mathcal{L}\left(\Gamma, p, B^{*}\right)$ is a compact 1-dimensional $C^{\infty}$ manifold with boundary. Moreover, $(t, \sigma)$ is a boundary point of $\mathcal{L}\left(\Gamma, p, B^{*}\right)$ if and only if either $\sigma_{j}^{i}=0$ for exactly one $s_{j}^{i} \in B^{*}$, or exactly one $s_{j}^{i} \notin B^{*}$ is a best response to $\sigma^{-i}$, or $t=0$, or $t=1$.

Proof Fix $n$ and $m^{i}, i \in N$. Fix a prior $p \in \Sigma$ and an admissible subset $B^{*}$ of $S^{*}$. The set $U$ consisting of $u \in \mathbb{R}^{m n}$ such that $g^{B^{*}, u} \pitchfork\{0\},\left(g^{B^{*}, u}, h_{j^{\prime}}^{B^{*}, u}\right) \pitchfork\{0\}$ for all $j^{\prime} \in\left\{1, \ldots, m^{*}+2\right\}$, and $\left(g^{B^{*}, u}, h_{j^{\prime}}^{B^{*}, u}, h_{j^{\prime \prime}}^{B^{*}, u}\right) \pitchfork\{0\}$ for all $j^{\prime}, j^{\prime \prime} \in\left\{1, \ldots, m^{*}+2\right\}$ with $j^{\prime} \neq j^{\prime \prime}$, has full measure by Lemmas A.1, A.2 and A.3.

Consider any $u \in U$. We show that $M\left[g^{B^{*}, u}, h^{B^{*}, u}\right]$ is a regular constraint set. Consider any $\bar{x} \in M\left[g^{B^{*}, u}, h^{B^{*}, u}\right]$. It has to be shown that

$$
\left\{\partial_{x} g_{j}^{B^{*}, u}(\bar{x}) \mid j \in J^{1}\right\} \cup\left\{\partial_{x} h_{j}^{B^{*}, u}(\bar{x}) \mid j \in J^{0}(\bar{x})\right\}
$$

is a set of independent vectors. When $J^{0}(\bar{x})=\emptyset$ this is a consequence of Lemma A.1, when $\left|J^{0}(\bar{x})\right|=1$ a consequence of Lemma A.2. Suppose $\left|J^{0}(\bar{x})\right| \geq 2$ and choose two elements, say $j^{\prime}$ and $j^{\prime \prime}$ from $J^{0}(\bar{x})$. It follows from Lemma A.3 that

$$
\left\{\partial_{x} g_{j}^{B^{*}, u}(\bar{x}) \mid j \in J^{1}\right\} \cup\left\{\partial_{x} h_{j^{\prime}, u}^{B^{*},}(\bar{x}), \partial_{x} h_{j^{\prime \prime}}^{B^{*}, u}(\bar{x})\right\}
$$

is a set of independent vectors, which leads to a contradiction as the set contains $2 m^{*}+n+2$ vectors of dimension $2 m^{*}+n+1$. Consequently, $\left|J^{0}(\bar{x})\right| \leq 1$, and $M\left[g^{B^{*}, u}, h^{B^{*}, u}\right]$ is a regular constraint set. It follows that $\mathcal{O}\left(\Gamma, p, B^{*}\right)$ is a 1-dimensional manifold with boundary, and that the boundary of $\mathcal{O}\left(\Gamma, p, B^{*}\right)$ is given by the elements $\bar{x} \in M\left[g^{B^{*}, u}, h^{B^{*}, u}\right]$ for which $\left|J^{0}(\bar{x})\right|=1$. Therefore, a point in $\mathcal{O}\left(\Gamma, p, B^{*}\right)$ is a boundary point if and only if exactly one of the inequalities in (5)-(8) holds with equality. These properties carry over to $\mathcal{L}\left(\Gamma, p, B^{*}\right)$ since it is diffeomorphic to $\mathcal{O}\left(\Gamma, p, B^{*}\right)$ by Theorem 3.1 .

Compactness of $\mathcal{L}\left(\Gamma, p, B^{*}\right)$ is obvious.

It remains to be shown that there is an open set of games and priors with full Lebesgue measure for which the asserted properties hold. Let $\Omega$ be the set of all noncooperative 
games an priors and let $\Omega^{B^{*}}$ be the subset of $\Omega$ consisting of all pairs $(\Gamma, p)$ for which the transversality statements in Lemma A.1, Lemma A.2 and Lemma A.3 hold. It has already been shown that $\Omega \backslash \Omega^{B^{*}}$ has Lebesgue measure zero. If $(\Gamma, p) \in \Omega \backslash \Omega^{B^{*}}$, then there exist $(t, \sigma, \lambda, \mu) \in \mathbb{R}^{2 m^{*}+n+1}$ such that $(t, \sigma, \lambda, \mu, \Gamma, p)$ belongs to the set $\Xi^{B^{*}}$ defined by

$$
\left.\begin{array}{rl}
\Xi^{B^{*}}= & \left\{(y, \Gamma, p) \in \mathbb{R}^{2 m^{*}+n+1} \times \Omega \mid\right. \\
& g^{B^{*}, u, p}(y)=0 \text { and } \operatorname{rank}\left(\partial g^{B^{*}, u, p}(y)\right) \leq 2 m^{*}+n-1 \\
& \exists j^{\prime}, g^{B^{*}, u, p}(y)=h_{j^{\prime}}^{B^{*}, u, p}(y)=0 \text { and } \operatorname{rank}\left(\begin{array}{l}
\partial g^{B^{*}, u, p}(y) \\
\partial h_{j^{\prime}}^{B^{*}, u, p}(y)
\end{array}\right) \leq 2 m^{*}+n \\
& \exists j^{\prime}, j^{\prime \prime}, j^{\prime} \neq j^{\prime \prime}, g^{B^{*}, u, p}(y)=h_{j^{\prime}, u, p}^{B^{*}}(y)=h_{j^{\prime \prime}}^{B^{*}, u, p}(y)=0
\end{array}\right\} .
$$

The notation $g^{B^{*}, u, p}$ and $h^{B^{*}, u, p}$ makes the dependence on the prior $p$ explicit. The set $\Xi^{B^{*}}$ is closed since it is obtained by finite unions and intersections of closed sets. We define the projection $\pi: \Xi^{B^{*}} \rightarrow \Omega$ by

$$
\pi(t, \sigma, \lambda, \mu, \Gamma, p)=(\Gamma, p) \text { for all }(t, \sigma, \lambda, \mu, \Gamma, p) \in \Xi^{B^{*}} .
$$

It holds that $\pi\left(\Xi^{B^{*}}\right)=\Omega \backslash \Omega^{B^{*}}$. We show that $\pi$ is proper, i.e., that $\pi^{-1}(K)$ is compact if $K$ is a compact subset of $\Omega$. Let $K$ be a compact subset of $\Omega$. Clearly, $\pi^{-1}(K)$ is a closed subset of the set $\Xi^{B^{*}}$, which is easily seen to be compact, and therefore $\pi^{-1}(K)$ is compact. Since the image by a continuous proper mapping of a closed set is closed, it follows that $\pi\left(\Xi^{B^{*}}\right)=\Omega \backslash \Omega^{B^{*}}$ is closed, so $\Omega^{B^{*}}$ is open. Since there is a finite number of admissible subsets $B^{*}$ of $S^{*}$, the intersection of the sets $\Omega^{B^{*}}$ over all admissible subsets $B^{*}$ is an open set of full Lebesgue measure of games and priors with the properties as stated in the theorem.

Theorem 3.4 For an open set of games $\Gamma \in \mathcal{G}$ and priors $p \in \Sigma$ with full Lebesgue measure, $\mathcal{L}(\Gamma, p)$ is a compact 1-dimensional piecewise $C^{\infty}$ manifold with boundaryy. The boundary of $\mathcal{L}(\Gamma, p)$ is given by the intersection of $\mathcal{L}(\Gamma, p)$ and $\{0,1\} \times \Sigma$. There is a unique boundary point in $\{0\} \times \Sigma$.

Proof Fix any game and any prior the open set of games $\Gamma \in \mathcal{G}$ and priors $p \in \Sigma$ with full Lebesgue measure of Theorem 3.2. For any $B^{*}$ it holds that $\mathcal{L}\left(\Gamma, p, B^{*}\right)$ is a compact 1dimensional $C^{\infty}$ manifold with boundary, so it consists of a finite number of arcs and loops. Each arc in $\mathcal{L}\left(\Gamma, p, B^{*}\right)$ has two endpoints. If it has an endpoint as in alternative (i) of Theorem 3.3, then it does not belong to any $\mathcal{L}\left(\Gamma, p, \bar{B}^{*}\right)$ for $\bar{B}^{*} \neq B^{*}$ and lies in $\{0,1\} \times \Sigma$. Therefore, it is a boundary point of $\mathcal{L}(\Gamma, p)$. If an arc has an endpoint as in alternative (ii) of Theorem 3.3, then it belongs to $(0,1) \times \Sigma$ and there exists exactly one $\bar{B}^{*} \neq B^{*}$ such 
that this endpoint is also an endpoint of an $\operatorname{arc}$ in $\mathcal{L}\left(\Gamma, p, \bar{B}^{*}\right)$. This $\operatorname{arc}$ in $\mathcal{L}\left(\Gamma, p, \bar{B}^{*}\right)$ has another endpoint that either satisfies alternative (i) of Theorem 3.3 or alternative (ii) of Theorem 3.3. In the former case an endpoint of $\mathcal{L}(\Gamma, p)$ in $\{0,1\} \times \Sigma$ has been found. In the latter case, the endpoint belongs to $(0,1) \times \Sigma$ and there is exists exactly one $\tilde{B}^{*} \neq \bar{B}^{*}$ such that this endpoint is also an endpoint of an $\operatorname{arc}$ in $\mathcal{L}\left(\Gamma, p, \tilde{B}^{*}\right)$, and so on.

Since there is a finite number of admissible subsets $B^{*}$ of $S^{*}$, and each $\mathcal{L}\left(\Gamma, p, B^{*}\right)$ consists of finitely many arcs and loops, it will either be the case that eventually an arc is generated with an endpoint as in alternative (i) of Theorem 3.3, or an arc is generated that has been generated before. In the latter case, we have found a piecewise $C^{\infty}$ loop of $\mathcal{L}(\Gamma, p)$. In the former case, the finite chain of arcs constitutes a piecewise $C^{\infty}$ arc of $\mathcal{L}(\Gamma, p)$ with endpoints belonging to $\{0,1\} \times \Sigma$. Indeed, starting from the endpoint already found in $\{0,1\} \times \Sigma$ and generating the chain of $\operatorname{arcs}$ in $\mathcal{L}\left(\Gamma, p, B^{*}\right)$ for various admissible subsets $B^{*}$, will either lead to another endpoint of $\mathcal{L}(\Gamma, p)$ in $\{0,1\} \times \Sigma$ or to the generation of an endpoint of some $\mathcal{L}\left(\Gamma, p, B^{*}\right)$ in $(0,1) \times \Sigma$ that has been generated before. The latter case is excluded as this point would be an endpoint of three different arcs, which violates Theorem 3.3. It follows that $\mathcal{L}(\Gamma, p)$ is a compact 1-dimensional piecewise $C^{\infty}$ manifold with boundary, where the boundary is given by the intersection of $\mathcal{L}(\Gamma, p)$ and $\{0,1\} \times \Sigma$. The reader might have observed that the argument to show this is nothing but a nonlinear version of the door-in door-out principle of Lemke and Howson (1964).

It remains to be shown that there is a unique boundary point in $\{0\} \times \Sigma$. Notice that $(0, \sigma) \in \mathcal{L}(\Gamma, p)$ if and only if for all $i, \sigma^{i}$ is a best response against the prior $p$. If there would be more than one best response for some player, then there would be at least two pure best responses for some player when $t=0$, which contradicts Lemma A.3.

Lemma A.4 For an open set of games $\Gamma \in \mathcal{G}$ and priors $p \in \Sigma$ with full Lebesgue measure, $\partial_{(t, \alpha, \mu)} \mathcal{H}(\bar{t}, \bar{\alpha}, \bar{\mu})$ has full rank for all $(\bar{t}, \bar{\alpha}, \bar{\mu})$ with $\mathcal{H}(\bar{t}, \bar{\alpha}, \bar{\mu})=0, \partial_{(\alpha, \mu)} \mathcal{H}(0, \bar{\alpha}, \bar{\mu})$ has full rank for all $(\bar{\alpha}, \bar{\mu})$ with $\mathcal{H}(0, \bar{\alpha}, \bar{\mu})=0$, and $\partial_{(\alpha, \mu)} \mathcal{H}(1, \bar{\alpha}, \bar{\mu})$ has full rank for all $(\bar{\alpha}, \bar{\mu})$ with $\mathcal{H}(1, \bar{\alpha}, \bar{\mu})=0$.

Proof Fix any game and any prior in the open set of games $\Gamma \in \mathcal{G}$ and priors $p \in \Sigma$ with full Lebesgue measure of Theorem 3.2. In Figure 8 the Jacobian of $\mathcal{H}$ is shown. To simplify the exposition of this Jacobian, the strategies $s_{j}^{i}$ are first ordered according to whether $\alpha_{j}^{i}$ is positive or not.

The Jacobian of $\mathcal{H}$ can be decomposed as the product of the two matrices shown in Figure 9. The first matrix equals the rows in (1) and (4) of Figure 7 when $B^{*}=\left\{s_{j}^{i} \in\right.$ $\left.S^{*} \mid \alpha_{j}^{i}>0\right\}$. Because $g^{B^{*}, u} \pitchfork\{0\}$, this matrix has full rank. In the second matrix, the notation $\Lambda(2 \alpha)$ is used to represent a diagonal matrix with the components of the vector 


\begin{tabular}{|c|c|c|c|c|c|}
\hline & $\partial_{t}$ & & & $\partial_{\mu}$ & \\
\hline & & $\alpha>0$ & $\alpha \leq 0$ & & \\
\hline \multirow{2}{*}{$m^{*}$} & \multirow{2}{*}{$\partial_{t} v(\cdot)$} & $\partial_{\sigma} v(\cdot) \partial_{\alpha} \sigma(\cdot)$ & 0 & \multirow{2}{*}{$-E^{\top}$} & $\alpha>0$ \\
\hline & & 0 & $\partial_{\alpha} \lambda(\cdot)$ & & $\alpha \leq 0$ \\
\hline$n$ & 0 & $E_{\alpha}$ & 0 & 0 & \\
\hline
\end{tabular}

where $E\left(i, s_{j^{\prime}}^{i^{\prime}}\right)=\left\{\begin{array}{ll}1 & \text { if } i=i^{\prime} \\ 0 & \text { otherwise }\end{array}\right.$ and $E_{\alpha}\left(i, s_{j^{\prime}}^{i^{\prime}}\right)= \begin{cases}2 \alpha_{j^{\prime}}^{i^{\prime}} & \text { if } i=i^{\prime} \\ 0 & \text { otherwise }\end{cases}$

Figure 8: The Jacobian of the homotopy function $\mathcal{H}$.

$2 \alpha$ on the diagonal. The second matrix contains at least $m^{*}$ zero rows. Since it cannot be the case that two variables $\alpha_{j}^{i}$ are zero at the same time, the second matrix contains at most $m^{*}+1$ zero rows.

\begin{tabular}{|c|c|c|c|c|c|c|}
\hline & $\partial_{t}$ & ${ }_{B^{*}} \partial_{S_{S^{*} \backslash B^{*}}}$ & $B^{*}$ & $\lambda_{S^{*} \backslash B^{*}}$ & $\partial_{\mu}$ & \\
\hline$m^{*}$ & $\partial_{t} v(\cdot)$ & $-\partial_{\sigma} v(\cdot)$ & $I$ & 0 & $-E^{\top}$ & $\begin{array}{l}B^{*} \\
C^{*}-D^{*}\end{array}$ \\
\hline$n$ & 0 & $E$ & & 0 & 0 & \\
\hline
\end{tabular}

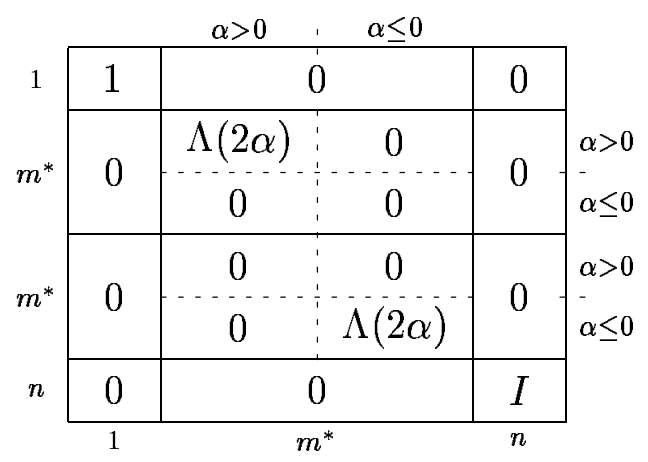

Figure 9: Decomposition of the Jacobian of $\mathcal{H}$ into two matrices.

We delete the $m^{*}$ zero rows in the middle part of the second matrix and the corresponding columns in the first matrix. The product of the resulting matrices remains equal to the Jacobian of $\mathcal{H}$. By deleting the zero rows in the second and the corresponding columns in the first matrix both matrices do not drop rank. For the second matrix this is obvious. For the first matrix, note that after deletion of the columns, it is equal to the submatrix of Figure 7 that results after applying Gaussian elimination to the rows in (1) and (4) by the rows in (2) and (3), and therefore has full rank.

If $\alpha_{j}^{i} \neq 0$ for all strategies $s_{j}^{i}$, then the remaining second matrix is a diagonal matrix with non-zero entries on the diagonal, so it has full rank. As a consequence, the product of the remaining matrices and thereby the Jacobian of $\mathcal{H}$ has full rank.

If $\alpha_{\ell}^{k}=0$ for a certain strategy $s_{\ell}^{k}$, then the remaining second matrix has still one zero row. After deletion of this row, the remaining second matrix still has full rank. After deletion of the corresponding column, the remaining first matrix also has full rank, as it is 
equal to the submatrix of Figure 7 that results after applying Gaussian elimination to the rows in (1) and (4) by the rows in (2) and (3), and the row in (6) corresponding to $s_{\ell}^{k}$. As the remaining first matrix is a square matrix, it follows that the product of the remaining matrices, and thereby the Jacobian of the homotopy function, has full rank. Consequently, $\partial_{(t, \alpha, \mu)} \mathcal{H}(\bar{t}, \bar{\alpha}, \bar{\mu})$ has full rank for all $(\bar{t}, \bar{\alpha}, \bar{\mu})$ with $\mathcal{H}(\bar{t}, \bar{\alpha}, \bar{\mu})=0$.

If we want to prove that $\partial_{(\alpha, \mu)} \mathcal{H}(0, \bar{\alpha}, \bar{\mu})$ has full rank for $(\bar{\alpha}, \bar{\mu})$ with $\mathcal{H}(0, \bar{\alpha}, \bar{\mu})=0$, and that $\partial_{(\alpha, \mu)} \mathcal{H}(1, \bar{\alpha}, \bar{\mu})$ has full rank for all $(\bar{\alpha}, \bar{\mu})$ with $\mathcal{H}(1, \bar{\alpha}, \bar{\mu})=0$, we have to consider the matrix in Figure 8 without the first column. That matrix can be decomposed as the product of the matrices of Figure 9 with the first column of the first matrix and both the first row and the first column of the second matrix deleted. The resulting second matrix contains exactly $m^{*}$ zero rows, as none of the variables $\alpha_{j}^{i}$ equals zero when $t=0$ or $t=1$. We delete the $m^{*}$ zero rows of the second matrix and the corresponding columns in the first matrix. The product of the resulting matrices remains equal to the Jacobian of $\mathcal{H}$. The resulting first matrix is equal to the submatrix of Figure 7 that is obtained after applying Gaussian elimination to the rows in (1) and (4) by the rows in (2) and (3), and the row in (7) when $t=0$ or the row in (8) when $t=1$. Therefore, both the resulting first matrix and the resulting second matrix are square matrices with full rank, and so is their product. Consequently, $\partial_{(\alpha, \mu)} \mathcal{H}(0, \bar{\alpha}, \bar{\mu})$ has full rank for all $(\bar{\alpha}, \bar{\mu})$ with $\mathcal{H}(0, \bar{\alpha}, \bar{\mu})=0$, and $\partial_{(\alpha, \mu)} \mathcal{H}(1, \bar{\alpha}, \bar{\mu})$ has full rank for all $(\bar{\alpha}, \bar{\mu})$ with $\mathcal{H}(1, \bar{\alpha}, \bar{\mu})=0$.

Theorem 4.1 For an open set of games $\Gamma \in \mathcal{G}$ and priors $p \in \Sigma$ with full Lebesgue measure, $\mathcal{H}^{-1}(\{0\})$ is a compact 1-dimensional $C^{1}$ manifold with boundary. The boundary of $\mathcal{H}^{-1}(\{0\})$ equals the intersection of $\mathcal{H}^{-1}(\{0\})$ and $\{0,1\} \times \mathbb{R}^{m^{*}} \times \mathbb{R}^{n}$. There is a unique boundary point in $\{0\} \times \mathbb{R}^{m^{*}} \times \mathbb{R}^{n}$.

Proof Fix any game and any prior in the open set of games $\Gamma \in \mathcal{G}$ and priors $p \in \Sigma$ with full Lebesgue measure of Theorem 3.2. Consider the following system of equalities and inequalities,

$$
\begin{aligned}
& \mathcal{H}(t, \alpha, \mu)=0 \\
& t \geq 0 \\
& -t+1 \geq 0
\end{aligned}
$$

The set of solutions to this system equals $\mathcal{H}^{-1}(\{0\})$. It is a regular constraint set by Lemma A.4 and is therefore a 1-dimensional $C^{1}$ manifold with boundary as described in the theorem. The compactness of $\mathcal{H}^{-1}(\{0\})$ follows trivially. The uniqueness of the boundary point in $\{0\} \times \mathbb{R}^{m^{*}} \times \mathbb{R}^{n}$ carries over from Theorem 3.4. 


\section{References}

Brown, D.J., P.M. DeMarzo, and B.C. Eaves (1996), "Computing Equilibria when Asset Markets are Incomplete," Econometrica, 64, 1-27.

Damme, E. van (1987), Stability and Perfection of Nash Equilibria, Springer-Verlag, New York.

Damme, E. van (1995), "Game Theory: The Next Stage," in A.P. Kirman, and L.A. Gérard-Varet (eds.) Economics Beyond the Millenium, Oxford University Press.

Elzen, A.H. van den, and A.J.J. Talman (1999), "An Algorithmic Approach towards the Tracing Procedure of Harsanyi and Selten," Games and Economic Behavior, 28, 130-145.

Garcia, C.B., C.E. Lemke, and H.J. Lüthi (1973), "Simplicial Approximation of an Equilibrium Point of Noncooperative N-Person Games," in T.C. Hu and S.M Robinson (eds.), Mathematical Programming, Academic Press, New York, 227-260.

Garcia, C.B., and W.I. Zangwill (1981), Pathways to Solutions, Fixed Points, and Equilibria, Prentice Hall, Englewood Cliffs.

Harsanyi, J.C. (1975), "The Tracing Procedure: A Bayesian Approach to Defining a Solution for $n$-Person Noncooperative Games," International Journal of Game Theory, 4, $61-94$.

Harsanyi, J.C., and R. Selten (1988), A General Theory of Equilibrium Selection in Games, MIT Press, Cambridge.

Herings, P.J.J. (1997a), "A Globally and Universally Stable Price Adjustment Process," Journal of Mathematical Economics, 27, 163-193.

Herings, P.J.J. (1997b), "Two Simple Proofs of the Feasibility of the Linear Tracing Procedure," CentER Discussion paper, No. 9777, Tilburg University. Forthcoming in Economic Theory.

Herings, P.J.J., and A.H. van den Elzen (1998), "Computation of the Nash Equilibrium Selected by the Tracing Procedure in N-Person Games," CentER Discussion paper, No. 9804, Tilburg University.

Herings, P.J.J., and K. Schmedders (1999), "Computing Equilibria in Finance Economies with Incomplete Markets and Transaction Costs," mimeo. 
Jongen, H.Th., P. Jonker, and F. Twilt (1983), Nonlinear Optimization in $\mathbb{R}^{n}$, I. Morse Theory, Chebyshev Approximation, Methoden und Verfahren der mathematische Physik, 29, Peter Lang, Frankfurt.

Judd, K.L. (1997), "Computational Economics and Economic Theory: Substitutes and Complements?," Journal of Economic Dynamics and Control, 21, 907-942.

Laan, G. van der, A.J.J. Talman, and L. van der Heijden (1987), "Simplicial Variable Dimension Algorithms for Solving the Nonlinear Complementarity Problem on a Product of Unit Simplices Using a General Labelling," Mathematics of Operation Research, 12, 377397.

Lemke, C.E., and J.T. Howson Jr. (1964), "Equilibrium Points of Bimatrix Games," SIAM Journal on Applied Mathematics, 12, 413-423.

Mas-Colell, A. (1985), The Theory of General Economic Equilibrium: A Differentiable Approach, Cambridge University Press, Cambridge.

McKelvey, R.D., and A. McLennan (1996), "Computation of Equilibria in Finite Games," in H.M. Amman, D.A. Kendrick, and J. Rust (eds.), Handbook of Computational Economics, Volume I, Elsevier Science.

McLennan, A. (1999), "The Expected Number of Nash Equilibria of a Normal Form Game," mimeo.

Rosenmüller, J. (1971), "On a Generalization of the Lemke-Howson Algorithm to Noncooperative $N$-Person Games," SIAM Journal on Applied Mathematics, 21, 73-79.

Schanuel, S.H., L.K. Simon, and W.R. Zame (1991), "The Algebraic Geometry of Games and the Tracing Procedure," in R. Selten (ed.), Game Equilibrium Models II, Methods, Morals and Markets, Springer-Verlag, Berlin, 9-43.

Watson, L.T. (1979), "A Globally Convergent Algorithm for Computing Fixed Points of $C^{2}$ Maps," Applied Mathematic and Computation, 5, 297-311.

Watson, L.T., and D. Fenner (1980), "Chow-Yorke Algorithm for Fixed Points or Zeros of $C^{2}$ Maps," ACM Transactions on Mathematical Software, 6, 252-260.

Watson, L.T., S.C. Billups, and A.P. Morgan (1987), "HOMPACK: A Suite of Codes for Globally Convergent Homotopy Algorithms," ACM Transactions on Mathematical Software, 13, 281-310. 
Wilson, R. (1971), "Computing Equilibria of N-Person Games," SIAM Journal on Applied Mathematics, 21, 80-87. 